\title{
EGF and a GSK3 Inhibitor Deplete Junctional E-cadherin and Stimulate Proliferation in the Mature Mammalian Ear
}

\author{
Mikolaj M. Kozlowski, ${ }^{1}$ @Mark A. Rudolf, ${ }^{1}$ and ${ }^{-}$Jeffrey T. Corwin ${ }^{1,2}$ \\ ${ }^{1}$ Department of Neuroscience, University of Virginia, School of Medicine, Charlottesville, Virginia 22908, and ${ }^{2}$ Department of Cell Biology, University of \\ Virginia, Charlottesville, Virginia 22908
}

\begin{abstract}
Sensory hair cell losses underlie the vast majority of permanent hearing and balance deficits in humans, but many nonmammalian vertebrates can fully recover from hearing impairments and balance dysfunctions because supporting cells (SCs) in their ears retain lifelong regenerative capacities that depend on proliferation and differentiation as replacement hair cells. Most SCs in vertebrate ears stop dividing during embryogenesis; and soon after birth, vestibular SCs in mammals transition to lasting quiescence as they develop massively thickened circumferential F-actin bands at their E-cadherin-rich adherens junctions. Here, we report that treatment with EGF and a GSK3 inhibitor thinned the circumferential F-actin bands throughout the sensory epithelium of cultured utricles that were isolated from adult mice of either sex. That treatment also caused decreases in E-cadherin, $\beta$-catenin, and YAP in the striola, and stimulated robust proliferation of mature, normally quiescent striolar SCs. The findings suggest that E-cadherin-rich junctions, which are not present in the SCs of the fish, amphibians, and birds which readily regenerate hair cells, are responsible in part for the mammalian ear's vulnerability to permanent balance and hearing deficits.
\end{abstract}

Key words: actin; e-cadherin; hair cell; inner ear; regeneration; vestibular

Significance Statement

Millions of people are affected by hearing and balance deficits that arise when loud sounds, ototoxic drugs, infections, and aging cause hair cell losses. Such deficits are permanent for humans and other mammals, but nonmammals can recover hearing and balance after supporting cells regenerate replacement hair cells. Mammalian supporting cells lose the capacity to proliferate around the time they develop unique, exceptionally reinforced, E-cadherin-rich intercellular junctions. Here, we report the discovery of a pharmacological treatment that thins F-actin bands, depletes E-cadherin, and stimulates proliferation in longquiescent supporting cells within a balance epithelium from adult mice. The findings suggest that high E-cadherin in those supporting cell junctions may be responsible, in part, for the permanence of hair cell loss in mammals.

\section{Introduction}

Over 400 million people live with disabling and permanent hearing deficits that commonly arise when sensory hair cells (HCs) are lost through exposure to loud sounds and ototoxic drugs, infections, or as the consequence of aging (Nadol, 1993; WHO Media Centre, 2019). Millions more are afflicted with balance

Received Oct. 31, 2019; revised Feb. 2, 2020; accepted Feb. 9, 2020.

Author contributions: M.M.K., M.A.R., and J.T.C. designed research; M.M.K. and M.A.R. performed research; M.M.K. analyzed data; M.M.K. wrote the first draft of the paper; M.M.K., M.A.R., and J.T.C. edited the paper; M.M.K., M.A.R., and J.T.C. wrote the paper.

This work was supported by National Institute on Deafness and Other Communication Disorders/National Institutes of Health R01 DC000200 to J.T.C. and National Institute on Deafness and Other Communication Disorders/ National Institutes of Health F30 DC016806 to M.A.R.

A U.S. Provisional Patent Application related to the research reported in this manuscript is currently pending (application number 62/951,996, filed December 20, 2019; patent applicant: Jeffrey T. Corwin; name of inventors: Jeffrey T. Corwin, Mark A. Rudolf, Mikolaj M. Kozlowski).

Correspondence should be addressed to Jeffrey T. Corwin at corwin@virginia.edu.

https://doi.org/10.1523/JNEUROSCI.2630-19.2020

Copyright $@ 2020$ the authors disorders caused by $\mathrm{HC}$ losses in the vestibular epithelia (Agrawal et al., 2009). Severe HC losses produce hearing and balance deficits in mammals and nonmammals alike, but nonmammals recover from deafening and imbalance within weeks via regeneration that depends on epithelial supporting cells (SCs) whose progeny differentiate as replacement HCs, as well as SCs that can directly convert into HCs without an intervening cell division (Corwin and Cotanche, 1988; Ryals and Rubel, 1988; Jones and Corwin, 1996; Golub et al., 2012; Scheibinger et al., 2018). Regenerative proliferation is limited in mammals because their SCs become quiescent as they mature (Hume et al., 2003; Davies et al., 2007; Gu et al., 2007; Oshima et al., 2007; Lu and Corwin, 2008; Burns et al., 2012a). Just what maturational changes restrict proliferation of mammalian SCs has remained unanswered.

Early in development, SCs in the inner ears of mammals resemble their nonmammalian counterparts, which readily proliferate and replace HCs after damage. However, as mammals mature, their vestibular SCs and the pillar and Deiters cells within 
the organ of Corti develop intercellular junctions with specializations that are not found in most nonmammalian SCs. At about the time when those specializations arise, mammalian SCs become proliferatively quiescent. The "parking brake hypothesis" (Burns and Corwin, 2013) suggests that proliferation is limited not only by downregulation of developmental growth pathways, which occurs in both mammals and nonmammals, but also by the unique features of mammalian SCs that emerge with their maturation. One candidate for such an inhibitory feature is the massive maturational reinforcement of circumferential F-actin bands at the adherens junctions of mammalian vestibular SCs (Burns et al., 2013). Another candidate is the E-cadherin that accumulates at the junctions between the maturing vestibular SCs. Less pronounced F-actin reinforcement and E-cadherin expression occur in pillar cells and Deiters cells of the maturing organ of Corti. Both aspects of junctional reinforcement strongly correlate with declines in the capacity for mammalian vestibular SCs to proliferate in response to exogenous mitogens or damage to the epithelium (Burns et al., 2008; Collado et al., 2011b). Neither occurs in SCs of fish, amphibians, or birds that readily replace HCs (Burns et al., 2013).

Here, we report that short-term culture with a mixture of seven factors (7F) (McLean et al., 2017) stimulated robust proliferation of SCs in utricles from neonatal mice but failed to stimulate proliferation in utricles from mice older than 1 week, even after HC loss. When utricles from such mice were cultured for longer durations, $7 \mathrm{~F}$ progressively thinned the F-actin bands in SCs throughout the macula and reduced E-cadherin in striolar SCs. These changes preceded a sharp increase in S-phase entry in the striola. Other experiments showed that two components of $7 \mathrm{~F}$ (EGF and the GSK3 inhibitor CHIR-99021) are responsible for these effects. When combined, EGF and CHIR-99021 thinned F-actin bands throughout the sensory epithelium, reduced levels of E-cadherin, apical $\beta$-catenin, and apical YAP just in the striolar SCs, and led to mitosis in the striola, even in utricles from adults. The findings are consistent with the parking brake hypothesis and suggest a model in which the maturational reinforcement of E-cadherin-rich SC junctions leads to the sequestration of $\beta$-catenin and YAP and the maintenance of SC quiescence, which contributes to the permanence of hearing and balance deficits in mammals.

\section{Materials and Methods}

Utricle dissection and organotypic culture. All animals were handled in accordance with protocols approved by the Animal Care and Use Committee at the University of Virginia. Swiss-Webster mice of either sex were obtained from Charles Rivers Laboratories. Three-day-old and younger mice were killed on ice, and mice older than $3 \mathrm{~d}$ were killed with carbon dioxide. After decapitation, each head was skinned and disinfected in ice-cold 70\% ethanol for $15 \mathrm{~min}$. Heads were next moved to ice-cold DMEM/F12 with HEPES (Invitrogen) where utricles were isolated from temporal bones and the roof, otoconia, otolithic membrane, and nerve were mechanically removed. The dissected organs contained the entire sensory epithelium, a small portion of the surrounding nonsensory epithelium, and the underlying stromal tissue. Utricles were next adhered to glass-bottom dishes (Mat-Tek) that were coated with CellTak (Corning), as described previously (Meyers and Corwin, 2007), and cultured in medium. Media were changed every $24 \mathrm{~h}$. To ablate HCs, 2 mu neomycin sulfate (Sigma Millipore) was added to the culture media for $24 \mathrm{~h}$ and then washed out with 3 rinses of media. To trace the incidence of S-phase entry, utricles were cultured with 5-ethynyl-2'deoxyuridine (Cayman Chemical) at a concentration of $2.5 \mu \mathrm{g} / \mathrm{ml}$. To interfere with the polymerization of microtubules, utricles were cultured with nocodazole (Sigma Millipore) at a concentration of $50 \mathrm{ng} / \mathrm{ml}$.
Preparation of culture media. All media were serum-free and composed of DMEM/F12/HEPES medium (Invitrogen) supplemented with N2 and B27 nutrients (Invitrogen), Glutamax (Invitrogen), and ciprofloxacin $(10 \mu \mathrm{g} / \mathrm{ml}$; Bayer). For preparation of EC and 7F media, we additionally used EGF (50 ng/ml; Invitrogen), bFGF (50 ng/ml; Stemfactor), IGF-1 ( $50 \mathrm{ng} / \mathrm{ml}$; Invitrogen), CHIR-99021 (3 $\mu \mathrm{M}$; LC Laboratories), valproic acid ( $1 \mathrm{~mm}$; Millipore), phospho-vitamin C (100 $\mu \mathrm{g} / \mathrm{ml}$; Sigma Millipore), and the inhibitor 616452 ( $2 \mu \mathrm{m}$; Calbiochem).

Immunohistochemistry. Utricles were fixed in 4\% PFA (Electron Microscopy Sciences) for $1 \mathrm{~h}$ at room temperature or in Glyofixx (Thermo Fisher Scientific) overnight at $4^{\circ} \mathrm{C}$. Fixed utricles were then washed with PBS/0.02\% Triton X-100 (PBST) solution and blocked at room temperature for $1 \mathrm{~h}$ in PBST containing either 10\% normal goat serum (Vector Laboratories) or 3\% normal donkey serum (Jackson ImmunoResearch Laboratories). Samples were next incubated overnight at $4{ }^{\circ} \mathrm{C}$ with appropriate primary antibodies, washed with PBST, and incubated with AlexaFluor-conjugated secondary antibodies (Invitrogen; 1:500) at room temperature for $2 \mathrm{~h}$. Then samples were washed with PBST and mounted on glass coverslips in ProLong Diamond Antifade Mountant. Images were acquired on a Zeiss 880LSM Airyscan confocal microscope.

Antibody characterization, EdU imaging, and F-actin labeling. To label SCs, we used rabbit anti-Sox2 (Cell Signaling Technology; 1:200; 23064S) and mouse IgG1 anti-Sox2 (Thermo Fisher Scientific; 1:100; MA1-014). To label HCs, we used mouse IgG1 anti-spectrin (Millipore; 1:50; MAB1622) and mouse IgG1 anti-Myo7a (Developmental Studies Hybridoma Bank; 1:50; 138-1). To image striolar HCs, we used goat antioncomodulin (Santa Cruz Biotechnology; 1:100; sc-7446) and rabbit anti-oncomodulin (Swant; 1:1000; OMG4). To image junctional proteins, we used rabbit anti-ZO-1 (Invitrogen; 1:200; 40-2200), mouse IgG1 anti-N-cadherin (BD Transduction Laboratories, 1:100; 610920), and mouse IgG2a anti-E-cadherin (BD Transduction Laboratories, $1: 200 ; 610181$ ), which recognizes the cytoplasmic domain of E-cadherin. We also used mouse IgG1 anti- $\beta$-catenin (BD Transduction Laboratories, $1: 400 ; 610153$ ) and mouse IgG2a anti-YAP (Santa Cruz Biotechnology, 1:50; sc-101199). To image cells in late $G_{2}$ and mitosis phases, we used mouse IgG1 anti-pH3 (Cell Signaling Technology, 1:200; 9706S). EdU-positive cells were imaged using the Click-iT EdU AlexaFluor- 488 and -647 Imaging Kits (Thermo Fisher Scientific). To label F-actin, utricles were incubated with phalloidin conjugated to AlexaFluor-488 nm (Invitrogen; 1:200; A12379).

Calculation of HC density. To measure and calculate mean HC density, utricles were fixed and immunostained with antibodies against spectrin, which is expressed in cuticular plates of HCs. Then, confocal image stacks were acquired at $20 \times$ magnification to visualize the entire macula. Counts of spectrin-positive cells from five $100 \times 100 \mu \mathrm{m}$ regions located in the anterolateral, anteromedial, central, posterolateral, and posteromedial regions of the utricle were tabulated. The mean densities of HCs were calculated by dividing the counts by the areas sampled.

Measurements of apical junction regions (AJRs). To measure widths of AJRs, high-magnification images were taken from lateral, striolar, and medial regions of each mouse utricle using an Axiovert 200M microscope (Carl Zeiss). Regions within each utricle were distinguished with the use of antibody against oncomodulin, which labels striolar HCs. Widths of AJRs were determined from the phalloidin label intensity along a perpendicular line across the homotypic SC-SC adherens junction. The measurement line spanned circumferential F-actin bands in the two cells that shared each junction as previously described (Burns et al., 2008). Heterotypic SC-HC junctions were not measured. To avoid measurement bias, a custom R script was used to identify the start and end of each AJR. We measured widths of 35-40 AJRs in each region (105-120 AJRs per utricle) and calculated the mean widths for each region and whole macula.

Code accessibility. $\mathrm{R}$ script for measurement of AJRs is available at Github (https://github.com/utricle/AJR-Width-Quantification).

Measurement of the E-cadherin, $N$-cadherin, $\beta$-catenin, and YAP immunostaining intensities. To measure peak intensities of E-cadherin, $\mathrm{N}$-cadherin, and $\beta$-catenin immunostaining, high-magnification images centered on the line of polarity reversal were taken with an LSM 880 confocal microscope (Carl Zeiss) to simultaneously capture portions of the lateral and striolar regions. We drew lines across 40 junctions of 
striolar SCs and 40 junctions of lateral SCs. To measure peak intensities of YAP immunostaining, we placed $1.5-\mu \mathrm{m}$-diameter ROIs within 40 striolar SCs and within 40 lateral SCs. Those measurements were used to calculate average intensities of immunostaining for E-cadherin, $\mathrm{N}$-cadherin, $\beta$-catenin, and YAP in striolar SCs and lateral extrastriolar SCs. Next, we normalized the immunoreactivity of each protein in the apical domain of striolar SCs to that of lateral extrastriolar SCs in the same utricle, producing a striolar/lateral intensity ratio (S/L ratio) to facilitate comparisons.

Quantification of macular area. For quantification of macular area, images of cultured utricles were acquired with an LSM 880 confocal microscope (Carl Zeiss) at 20× magnification, and Sox 2 staining was used to delineate the border of the sensory epithelium. The area inside each outlined region was calculated using ImageJ.

Experimental design and statistical analysis. The experimental design is illustrated in the figures and described in figure legends. Images were carefully selected to show the average effect obtained for each experimental condition. All descriptive statistics are presented as mean \pm SD. For statistical comparisons, Microsoft Excel was used to conduct two-sample, two-tailed $t$ tests, and GraphPad Prism 8 software was used to conduct ANOVA and post hoc pairwise comparisons. Each utricle was considered an independent observation for statistical analysis. Animals of either sex were used. $p$ values $<0.05$ were considered statistically significant. No methods were used for sample size determination.

\section{Results}

A mixture of seven factors stimulates robust $S$-phase entry in utricles from newborn mice, but responses wane postnatally

McLean et al. (2017) induced significant proliferation in $3 \mathrm{D}$ cultures of cells that were dissociated from the neonatal mouse cochlea and cultured in a medium supplemented with seven factors (EGF, bFGF, IGF-1, phosphorylated vitamin $\mathrm{C}$, and small molecules that inhibit GSK3, HDAC, and TGF $\beta$ RI) (Fig. 1a). To test whether that medium, which we call " $7 \mathrm{~F}$," would overcome the age-related decline in the capacity for mammalian SCs to enter the cell cycle (Hume et al., 2003; Davies et al., 2007; Gu et al., 2007; Oshima et al., 2007; Lu and Corwin, 2008; Burns et al., 2012a), we dissected utricles from mice of different ages (P0, P2, P4, P6, P8, P16, and 6 weeks) and cultured them in either $7 \mathrm{~F}$ or control medium and EdU for $72 \mathrm{~h}$ (Fig. $1 b, c)$. SCs were identified by labeling with an antibody to Sox2.

The $\mathrm{P} 0$ utricles that were cultured in $7 \mathrm{~F}$ contained 8.6-fold more $\mathrm{EdU}^{+} / \mathrm{Sox} 2^{+}$ cells than utricles cultured in the control
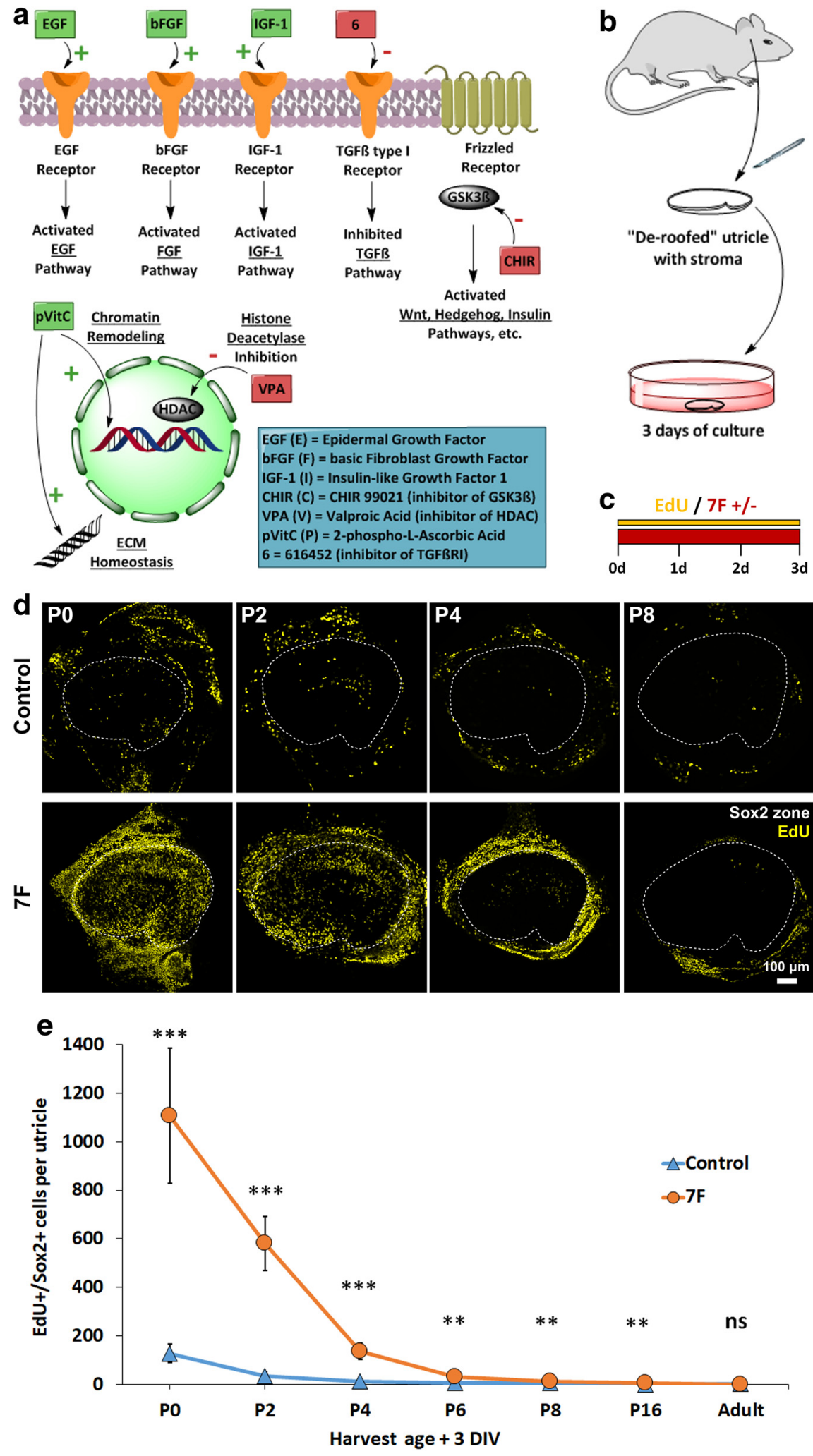

Figure 1. Three days in $7 F$ medium induced robust $S$-phase entry in organ cultures of undamaged utricles from newborn (PO) mice, but not in utricles from 8-d-old (P8) mice. $\boldsymbol{a}$, Signaling pathways affected by the 7F medium. $\boldsymbol{b}$, Utricles were dissected from mice of different ages and cultured after removal of the roof and otolithic membrane. $c$, Utricles were cultured for $3 \mathrm{~d}$ in the presence of EdU in medium with or without 7F. $\boldsymbol{d}$, Low-magnification images of robust S-phase entry in utricles from the youngest neonates, a response to the $7 \mathrm{~F}$ medium that declined sharply in utricles from older mice. $\boldsymbol{e}$, Quantification of EdU ${ }^{+} / \mathrm{Sox2}{ }^{+} \mathrm{SCs}$ in mouse utricles cultured $3 \mathrm{~d}$ in control medium (blue) and $7 \mathrm{~F}$ medium (orange) ( $n=7$ for P0, P2, P4, and $n=6$ for P6, P8, P16, Adult for Control and 7F). Data are mean \pm SD. ns, Not significant $(p \geq 0.05) .{ }^{* *} p<0.01,{ }^{* * *} p<0.001$. ECM, extracellular matrix; DIV, days in vitro. 
a
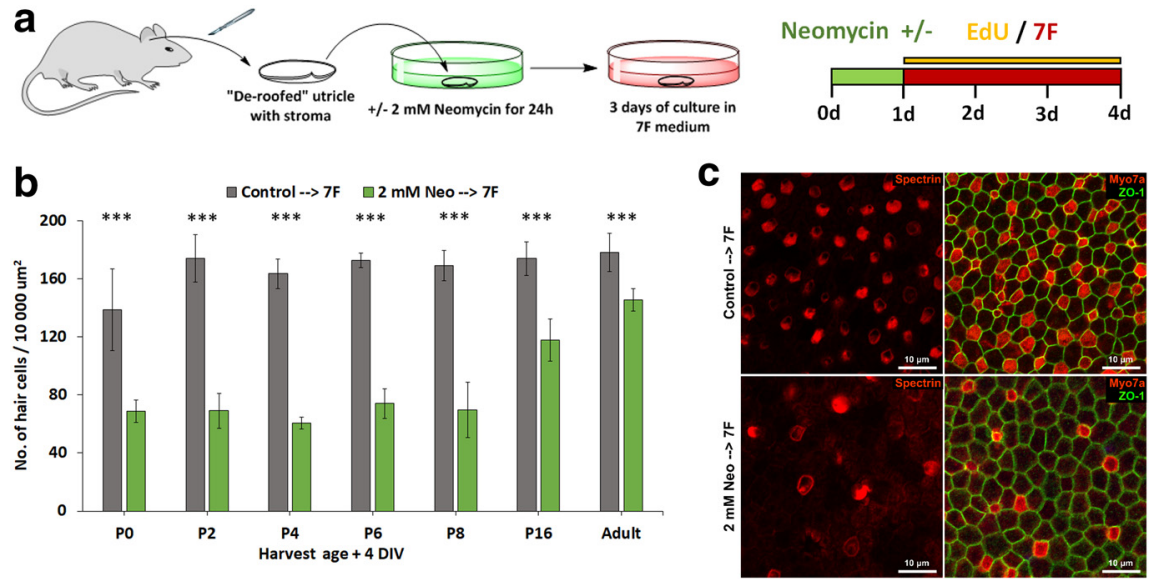

d
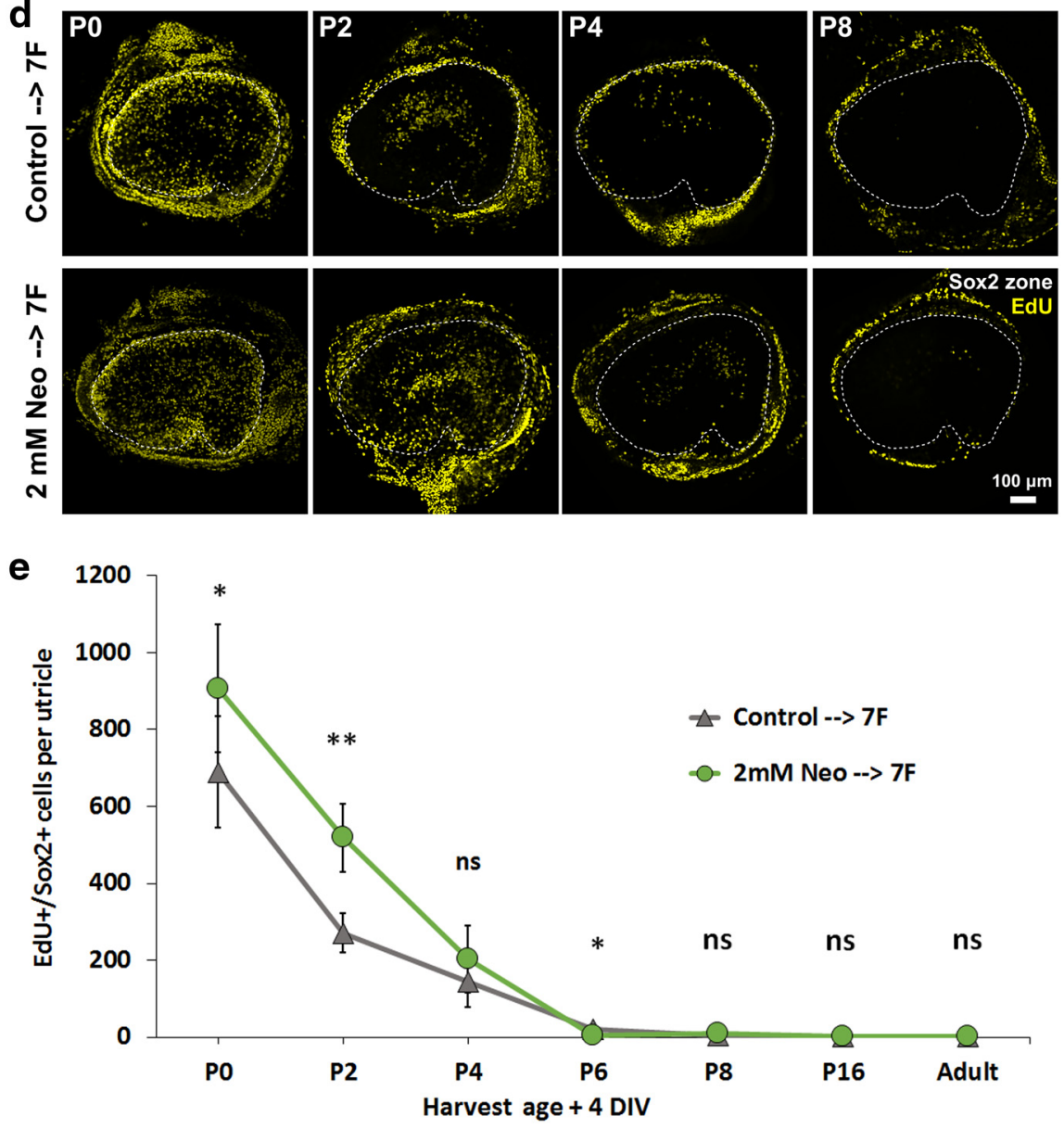

Figure 2. $\mathrm{HCl}$ loss increased the amount of S-phase entry in utricles from the youngest neonates but did not extend the age of responsiveness beyond P8. $\boldsymbol{a}$, Utricles were cultured in control medium or that medium plus 2 mm neomycin for $24 \mathrm{~h}$. Then, they were all cultured for $3 \mathrm{~d}$ in $7 \mathrm{~F}$ medium with EdU. $\boldsymbol{b}$, Quantification of HC density in utricles cultured under the two conditions ( $n=$ 8 for P0, $n=7$ for P2 and P4, and $n=6$ for P6, P8, P16, Adult for Control and 7F). c, Representative high-magnification images of $\mathrm{P} 2$ mouse utricles exposed to the $24 \mathrm{~h}$ pretreatment with $2 \mathrm{~mm}$ neomycin or control medium followed by $72 \mathrm{~h}$ in $7 \mathrm{~F}$ medium and EdU. HCs were labeled with antibodies against myosin VIIA and spectrin. $\boldsymbol{d}$, Low-magnification images show that experimental induction of $\mathrm{HC}$ loss potentiated S-phase entry in utricles from $\mathrm{PO}$ and $\mathrm{P} 2$ mice cultured in $7 \mathrm{~F}$ medium but had no such effect in utricles from mice older than 1 week. e, Quantification of EdU ${ }^{+} / \mathrm{Sox}{ }^{+} \mathrm{SC}$ in neomycin-treated and control utricles cultured $4 \mathrm{~d}$ ( $n=8$ for P0, $n=7$ for P2 and P4, and $n=6$ for P6, P8, P16, Adult for Control and 7F). The difference in P6 utricles was statistically significant, but with the $\mathrm{EdU}^{+} / \mathrm{Sox2}{ }^{+}$cells in control cultures exceeding the number of such cells in neomycin-treated cultures (control: $20 \pm 8$ vs neo: $5 \pm 2, p=0.01257, n=6$ ). Data are mean \pm SD. ns, Not significant $(p \geq 0.05) .{ }^{*} p<0.05,{ }^{* *} p<0.01$, *** $p<0.001$. DIV, days in vitro. medium (1107 \pm 279 vs $128 \pm 39$, respectively, mean $\pm \mathrm{SD} ; p=0.00008$, twotailed $t$ test, $n=7$ or 8 ), showing that 7F's stimulatory effects extend even to SCs in undamaged vestibular epithelia. As mice grew older, the incidence of $\mathrm{EdU}^{+} / \mathrm{Sox} 2^{+}$ cells declined quite sharply. The 7F culture protocol yielded $581 \pm 111 \mathrm{EdU}^{+} /$ Sox $2^{+}$cells in utricles harvested on $\mathrm{P} 2$, and $136 \pm 34$ and $11 \pm 4 \mathrm{EdU}^{+} / \mathrm{Sox} 2^{+}$ cells in utricles harvested from $\mathrm{P} 4$ and $\mathrm{P} 8$ mice, respectively (Fig. 1d,e). While the $7 \mathrm{~F}$ mixture evoked robust cell cycle entry of SCs in utricles of newborn mice, the $72 \mathrm{~h}$ exposure to activators of the EGF, bFGF, IGF-1, and Wnt pathways combined with inhibitors of HDAC and the TGF $\beta$ pathway failed to evoke substantial proliferation in utricles from older neonates, highlighting the dominant nature of quiescence that occurs with the postnatal maturation of mammalian SCs (Fig. 1e).

\section{HC loss does not restore proliferation in older utricles}

To test whether HC loss would increase $\mathrm{SC}$ responses in older ears, we dissected utricles from P0, P2, P4, P6, P8, P16, and 6 -week-old mice and cultured them for $24 \mathrm{~h}$ in control medium with or without 2 $\mathrm{mM}$ neomycin. After 3 rinses in control medium, all utricles were cultured for 3 more days in 7F and EdU (Fig. 2a). In the neomycin-treated utricles from $\mathrm{P} 8$ and younger mice, counts of spectrin-labeled cuticular plates showed a $50 \%-65 \%$ decrease in the average spatial density of HCs compared with the age-matched utricles cultured without neomycin, and HC density decreased 32\% and 19\% in the neomycin-treated utricles from P16 and 6-week-old mice, respectively (Fig. 2b,c). As expected, the loss of HCs significantly increased the numbers of $\mathrm{EdU}^{+} / \mathrm{Sox} 2^{+}$ cells in the utricles from $\mathrm{P} 0$ and $\mathrm{P} 2$ mice (P0 neo: $907 \pm 167$ vs P0 control: $690 \pm$ $144 ; p=0.01481$, two-tailed $t$ test, $n=8$; P2 neo: $519 \pm 89$ vs P2 control: $271 \pm 50$, $p=0.00773$, two-tailed $t$ test, $n=7$ ). However, the loss of HCs did not significantly increase the incidence of $\mathrm{EdU}^{+} /$ Sox $2^{+}$cells in the utricles from P4 and older mice (P4 control $145 \pm 67$ vs P4 neo $203 \pm 86, p=0.18728, n=7$; P6 control $20 \pm 8$ vs P6 neo $5 \pm 2, p=0.01257, n=$ 6; P8 control $5 \pm 4$ vs P8 neo $9 \pm 4, p=$ 0.13777, $n=6$; P16 control $1 \pm 1$ vs P16 neo $1 \pm 1, p=0.71659, n=6$; Adult control $1 \pm 1$ vs Adult neo $1 \pm 1, p=$ 0.87938, $n=6$, two-tailed $t$ tests) (Fig. $2 d)$. Indeed, after $3 \mathrm{~d}$ in $7 \mathrm{~F}$ and EdU, $\mathrm{EdU}^{+} /$Sox $2{ }^{+}$cells were rarely observed in either the treated or control utricles from 
P6, P8, P16, and 6-week-old mice (Fig. 2e). The fact that HC loss failed to restore the capacity for SCs to enter the cell cycle after a $3 \mathrm{~d}$ exposure to 7F suggests that the damage-evoked responses, which potentiate proliferation of P0 SCs, cannot surmount the age-related changes that prevent quiescent SCs from transitioning into a proliferative state.

\section{F thins F-actin bands in utricles from P8 mice}

The preceding results were consistent with the hypothesis that maturational changes somehow limit the proliferative capacity of mammalian SCs. One of the most prominent known maturational changes is the exceptional postnatal thickening of the circumferential bands of F-actin that bracket the SC-SC junctions in mammalian vestibular organs and in the Deiters and pillar cells in the organ of Corti (Meyers and Corwin, 2007; Burns et al., 2008, 2013). In contrast to mammalian ears, the circumferential F-actin bands in nonmammalian SCs, which readily replace HCs, remain thin throughout life (Burns et al., 2013). In a pilot study, we incidentally observed that culture in 7F appeared to result in thinning of the F-actin bands. To explore that possibility, we conducted an expanded investigation to test the hypothesis that culture in 7F reduces the thickness of the F-actin bands in mammalian vestibular SCs over time.

Since SCs in the utricles of P8 mice have thickened F-actin bands (Burns et al., 2008) and rarely entered the cell cycle during a 72 h culture with 7F (Fig. 1), groups of P8 utricles were cultured in either 7F or control medium and fixed after 1,2, 3, 4, or $5 \mathrm{~d}$. For comparison, we fixed utricles from P8 mice at the time of dissection. After staining with AlexaFluor phalloidin and antibodies against oncomodulin, a calcium-binding protein expressed in striolar HCs, we acquired images from standardized regions in the lateral, striolar, and medial sections of each utricle and measured the widths of AJRs, which span the F-actin bands in a pair of adjacent SCs and the intercellular junction those cells share (Burns et al., 2008).

In P8 utricles that were fixed immediately after dissection (time $=0 \mathrm{~d}$ ), the AJRs averaged $2.26 \pm 0.12 \mu \mathrm{m}$ (Fig. $3 a$ ). During the first $3 \mathrm{~d}$ of culture in $7 \mathrm{~F}$, we observed progressive thinning of the SC's F-actin bands $(0.40 \mu \mathrm{m} / \mathrm{d})$, resulting in a halving of the AJR width. At that point, AJR widths averaged $1.09 \pm 0.07 \mu \mathrm{m}$, similar to the average width of AJRs for SCs in P0 utricles in vivo $(1.12 \mu \mathrm{m})$ (Burns et al., 2008) (Fig. 3b). After that, the thinning effects of $7 \mathrm{~F}$ slowed $(0.04 \mu \mathrm{m} / \mathrm{d})$ and widths stabilized at $\sim 1.0$ $\mu \mathrm{m}$. Regional analysis showed that AJRs of striolar SCs were slightly thinner at the start of culture (0 DIV) and thinned more quickly than those of the lateral and medial extrastriolar SCs (Fig. $3 c$ ). The width of AJRs in utricles cultured in control medium for $3 \mathrm{~d}$ decreased by $17 \%(0.12 \mu \mathrm{m} / \mathrm{d}$ to an average width of $1.87 \pm$ $0.21 \mu \mathrm{m})$. The results showed that one or more of the constituents of 7F led to partial dismantling of the robust F-actin bands that are found in the SCs of mammalian ears, which thicken as the proliferative capacity of those SCs declines.

\section{Culture in 7F reduces E-cadherin in striolar SC junctions}

The SCs in vestibular and auditory epithelia of most nonmammalian ears express E-cadherin at very low levels or levels below normal detectability (Warchol, 2007; Burns et al., 2013). In contrast, the junctions between SCs in mammalian vestibular epithelia and those between inner and outer pillar SCs and the three rows of Deiters cells in the organ of Corti express considerable E-cadherin at levels that increase postnatally (Hackett et al., 2002; Collado et al., 2011b; Burns et al., 2013). In mouse utricles, junctional E-cadherin in SCs increases $>2.5$-fold between P1 and P16 and >6-fold between P1 and
P82, extending down the basolateral membranes (Collado et al., 2011b). Since 7F thinned the circumferential F-actin bands at the SC-SC junctions in cultured utricles, we asked whether it also would reduce the levels of E-cadherin.

To address that question, we cultured $\mathrm{P} 8$ utricles in either $7 \mathrm{~F}$ or control medium, fixed them after 1,3 , or $5 \mathrm{~d}$, and immunostained them with antibodies against E-cadherin, oncomodulin, and $\mathrm{N}$-cadherin. $\mathrm{N}$-cadherin is expressed in all HC-SC and SC-SC junctions and delineated the sensory epithelium. We observed no noticeable changes in the intensity of junctional E-cadherin immunostaining in the utricles that were cultured up to $5 \mathrm{~d}$ in control medium, but the level of E-cadherin immunostaining in utricles cultured in 7F decreased progressively in the striola while appearing to remain unchanged in the extrastriolar region (Fig. 4a). To quantify this, we acquired stacks of highmagnification, confocal images centered on the line of polarity reversal, which runs between the striolar and lateral regions of the macula ( $\mathrm{Li}$ et al., 2008), and calculated the S/L ratios of E-cadherin and N-cadherin intensity (Fig. $4 b$; see Materials and Methods). For the utricles cultured in control medium, $\mathrm{S} / \mathrm{L}$ ratios for E-cadherin ranged between 0.95 and 0.98 , independent of culture duration. In the utricles cultured in 7F, E-cadherin decreased in the striolar SCs and S/L ratios for E-cadherin declined progressively. After $3 \mathrm{~d}$ in $7 \mathrm{~F}$, the E-cadherin S/L ratio was significantly lower $(0.59 \pm 0.09$ vs $0.95 \pm 0.06$ in the controls; $p=$ 0.00050 , two-tailed $t$ test, $n=4)$ and decreased to $0.44 \pm 0.06$ after $5 \mathrm{~d}$ in $7 \mathrm{~F}$ ( 0.98 in controls; $p=0.00001$, two-tailed $t$ test, $n=$ 4) (Fig. 4d). There were no noticeable changes in the intensity of $\mathrm{N}$-cadherin immunostaining (Fig. $4 c$ ), and the S/L ratios for $\mathrm{N}$-cadherin did not differ significantly among the utricles cultured in 7F or control medium for 1,3 , or $5 \mathrm{~d}$ (1 DIV: $p=$ 0.48887, two-tailed $t$ test, $n=4 ; 3$ DIV: $p=0.39816$, two-tailed $t$ test, $n=4 ; 5$ DIV: $p=0.42423$, two-tailed $t$ test, $n=4)$ (Fig. $4 e$ ). Thus, 7F appeared to selectively reduce junctional E-cadherin in striolar SCs, whereas levels of N-cadherin remained unchanged.

\section{After their F-actin bands thin and E-cadherin decreases, striolar SCs enter S-phase}

In light of the preceding results, we decided to extend the culturing of utricles to determine whether a longer exposure to $7 \mathrm{~F}$ would increase S-phase entry. In these experiments, we cultured P8 utricles in 7F or control medium for various durations and added EdU for the final $24 \mathrm{~h}$, which allowed us to assess the kinetics of S-phase entry over the third, fourth, fifth, sixth, and seventh day of culture (Fig. $5 a$ ).

The number of EdU ${ }^{+} /$Sox $2^{+}$cells never exceeded 3 in any of the P8 utricles cultured in control medium, independent of the duration. The numbers of Sox $2^{+}$cells that labeled with EdU also remained relatively low on the third and fourth days of culture in $7 \mathrm{~F}$, consistent with our earlier results ( $3 \mathrm{~d}: 10 \pm 8,4 \mathrm{~d}$ : $12 \pm 7$; Fig. 1 e). On the fifth day of culture, the number of $\mathrm{EdU}^{+} / \mathrm{Sox} 2^{+}$cells increased $\sim 5$-fold ( $55 \pm 34$ ) and rose further on the sixth and seventh day $\left(83 \pm 19\right.$ and $77 \pm 48$, respectively). Virtually all of the Sox ${ }^{+}$ cells that had incorporated EdU on the fifth, sixth, and seventh day of culture in 7F were in the striola, which the oncomodulin labeling of HCs delineated (Fig. $5 c, d$ ). SCs that transitioned from quiescence to $\mathrm{S}$-phase on the fifth day and later in $7 \mathrm{~F}$ were restricted to the striola, where the levels of E-cadherin began to decline after $3 \mathrm{~d}$.

When delaminated utricular epithelia from rodents are maintained in direct contact with rigid substrates in prolonged culture, that can result in tissue spreading and increased S-phase entry (Gu et al., 2007). To control for that, we measured the areas of the sensory epithelium in P8 utricles that were cultured in $7 \mathrm{~F}$ or 
a
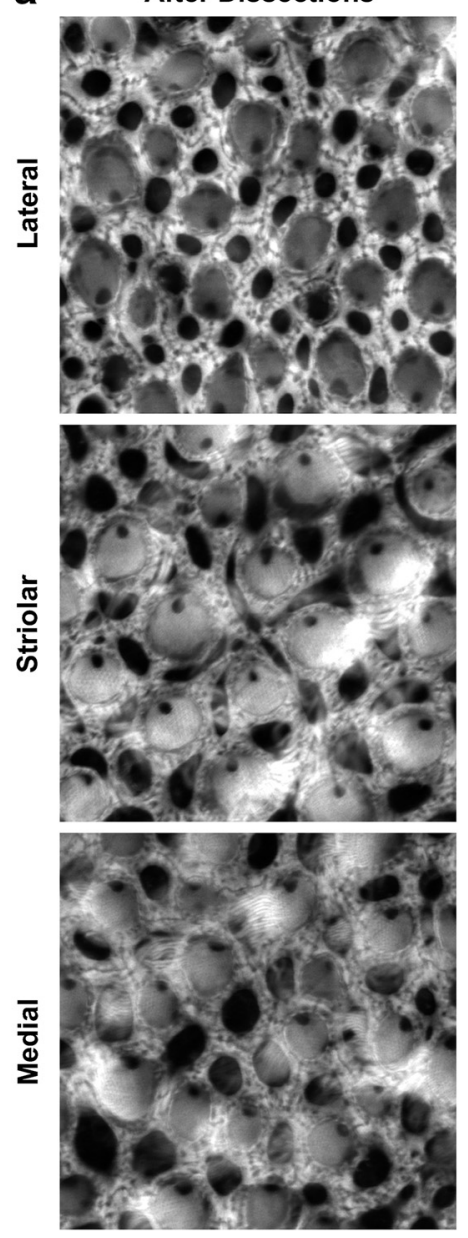

b

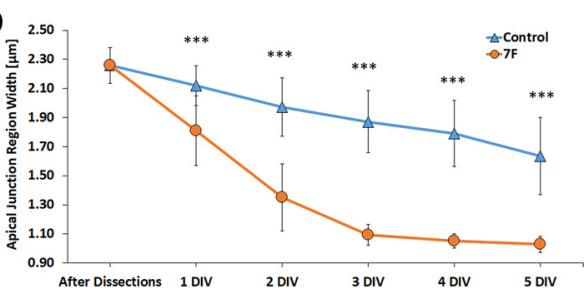

3 DIV in Control
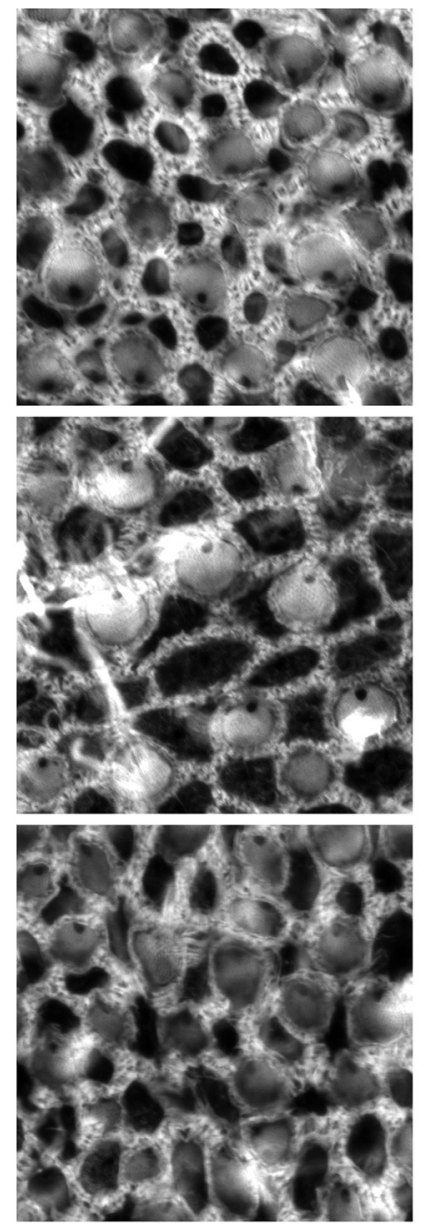

C

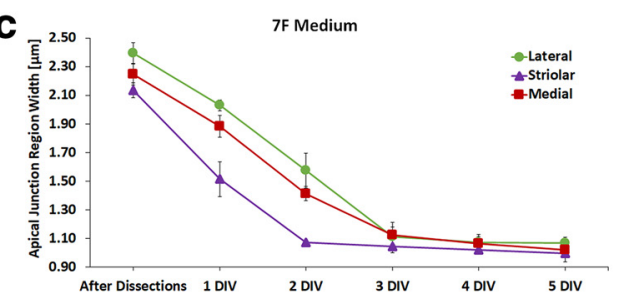

Figure 3. Culture in $7 \mathrm{~F}$ medium dismantled the thick circumferential F-actin bands that bracket SC junctions throughout all regions of the P8 mouse utricle. The thick F-actin bands are a unique feature of mature $\mathrm{SC}$ in the vestibular and cochlear epithelia of mammals. The thickening occurs early in postnatal maturation and is highly correlated with the age-related decline in the capacity for SCs to reenter the cell cycle. $\boldsymbol{a}$, Representative high-magnification images show visibly thinner F-actin bands in the lateral extrastriolar, striolar, and medial extrastriolar regions of P8 mouse utricles that were cultured in $7 \mathrm{~F}$ medium. $\boldsymbol{b}$, The width of AJRs progressively decreased in the control medium, but more quickly and substantially decreased during the first $3 \mathrm{~d}$ in the $7 \mathrm{~F}$ medium, plateauing at $\sim 1 \mu \mathrm{m}$ ( $n=4$ at each investigated time point for Control and 7F). $c$, AJR widths decreased faster in the striola than in the lateral and medial extrastriolar regions of the sensory epithelium ( $n=4$ at each investigated time point). Data are mean \pm SD. ns, Not significant $(p \geq 0.05)$. ${ }^{* *} p<0.001$. DIV, days in vitro.

control medium for 3, 4, 5, 6, and $7 \mathrm{~d}$. The mean area measurements were slightly, but not significantly, greater for utricles that were cultured in 7F compared with controls (3 DIV: $p=0.35597$, two-tailed $t$ test, $n=6$; 4 DIV: $p=0.07991$, two-tailed $t$ test, $n=$ 6; 5 DIV: $p=0.18047$, two-tailed $t$ test, $n=6 ; 6 \mathrm{DIV}: p=0.45152$, two-tailed $t$ test, $n=6$; 7 DIV 7 DIV: $p=0.77690$, two-tailed $t$ test, $n=6$ ), but areas did not increase with time in culture, consistent with the expected absence of spreading in the semiintact utricle cultures used here (Fig. 5b).

EGF and the GSK3 inhibitor CHIR-99021 thin F-actin bands, decrease E-cadherin, and induce striolar SCs to enter S-phase To determine which factors in 7F were responsible for thinning the F-actin bands and inducing the striolar SCs to transition from quiescence to the cell cycle, we cultured P8 utricles in seven different media. Each was supplemented with just one of the factors from the 7F mixture: EGF, bFGF, IGF-1, CHIR-99021, valproic acid, phosphorylated vitamin C, or 616452 (E/F/I/C/V/P/6). After $3 \mathrm{~d}$, we fixed each sample, labeled F-actin with AlexaFluor phalloidin, and measured the widths of AJRs in the lateral, striolar, and medial regions as in earlier experiments.

Only the medium that contained EGF (E) and the medium that contained CHIR-99021 (C) resulted in significant thinning of the AJRs between SCs in the P8 utricles (E: $p<0.0001$, ANOVA and then post hoc $t$ test, $n=4$; C: $p<0.0001$, ANOVA and then post hoc $t$ test, $n=4$ ) (Fig. $6 a$ ). The average width of AJRs decreased by $40 \%$ (to $1.36 \pm 0.19 \mu \mathrm{m}$ ) in the EGF medium and $34 \%$ (to $1.50 \pm 0.18 \mu \mathrm{m}$ ) in the CHIR-99021 medium. The 

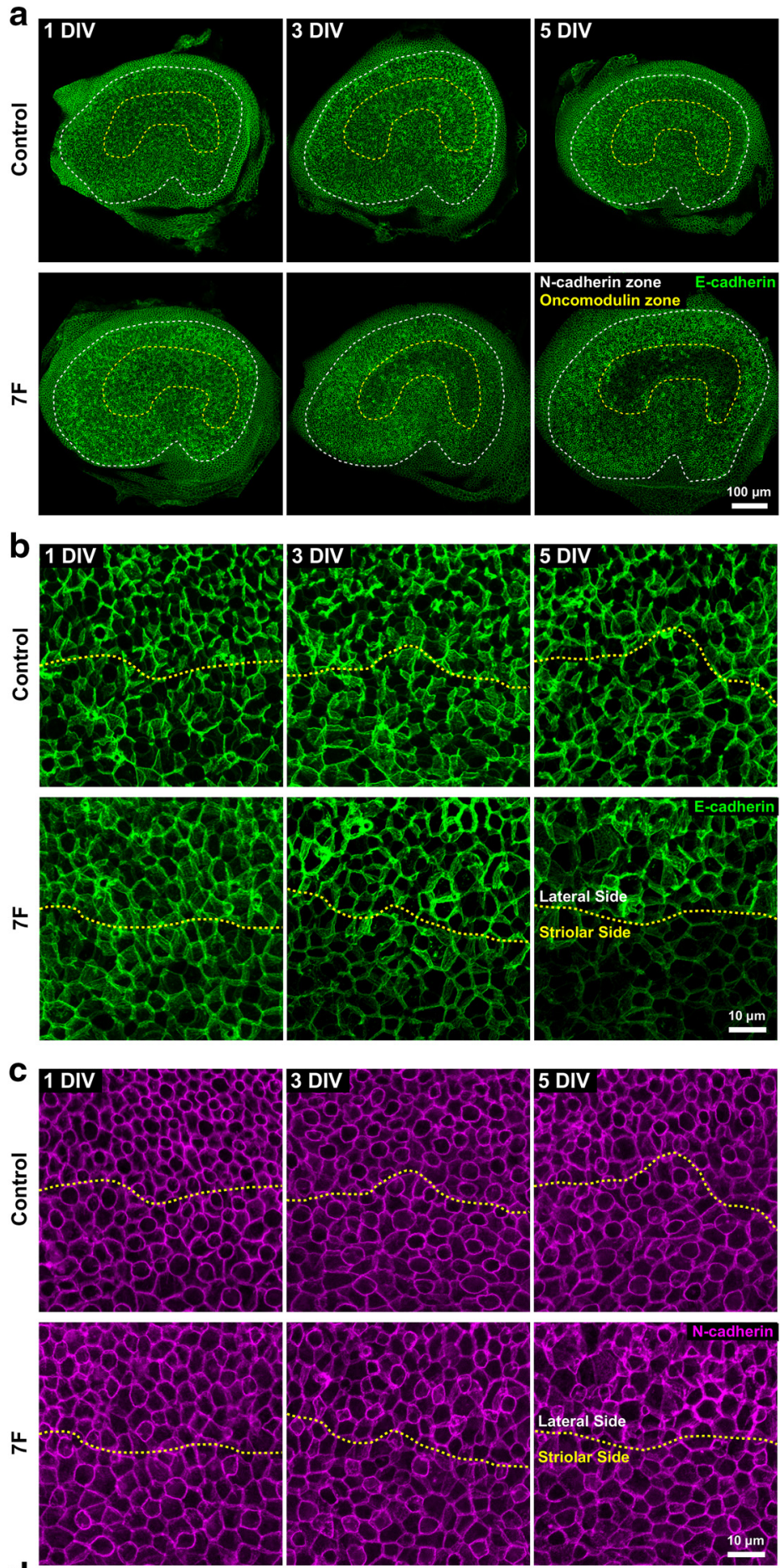

d

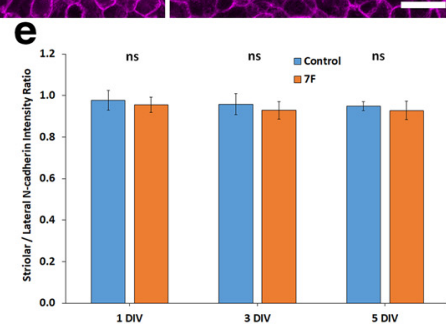

Figure 4. Prolonged culture of utricles from $\mathrm{P} 8$ mice in $7 \mathrm{~F}$ medium led to decreased levels of E-cadherin, but not $\mathrm{N}$-cadherin, at the junctions between SCs in the striola. $\boldsymbol{a}, \mathrm{E}$-cadherin immunoreactivity decreased in the striola of P8 mouse utricles cultured in $7 \mathrm{~F}$ medium, but not in those cultured in control medium. $\boldsymbol{b}$, High-resolution images centered on the line of polarity reversal (yellow dashes) depicting the decrease in E-cadherin at cell junctions. c, High-resolution images of N-cadherin immunostaining centered on the line of polarity reversal (yellow dashes). Levels of $\mathrm{N}$-cadherin remained unchanged during culture in $7 \mathrm{~F}$ and control medium. widths of AJRs in other media decreased modestly and similarly to the AJRs in utricles cultured in control medium. We then cultured P8 utricles in medium that contained both EGF and CHIR-99021 (EC medium). After $3 \mathrm{~d}$, the average width of AJRs decreased by $50 \%$, reaching $1.14 \pm 0.09 \mu \mathrm{m}(p<0.0001$, ANOVA and then post hoc $t$ test, $n=4$ ), which was significantly lower than AJR widths in utricles cultured in $\mathrm{E}$ or $\mathrm{C}$ alone $(p=$ 0.00215 and $p=0.00001$, respectively, two-tailed $t$ test, $n=4$ ) (Fig. 6a,b). Earlier, we found that treatment with complete 7F decreased the average width of AJRs by $52 \%$ (to $1.09 \pm 0.07 \mu \mathrm{m}$ ). The similarity suggests that EGF and CHIR-99021 are largely responsible for 7F's thinning of the F-actin bands.

Next, we tested whether culture in EC medium would reduce E-cadherin in the striolar SC junctions. For this, P8 utricles were cultured in EC medium for $5 \mathrm{~d}$, then fixed and immunostained with antibodies against $\mathrm{E}$-cadherin, $\mathrm{N}$-cadherin, and oncomodulin. In those utricles, the $\mathrm{S} / \mathrm{L}$ ratio for E-cadherin was significantly reduced to $0.55 \pm 0.04(p<0.00001$, two-tailed $t$ test, $n=4$ ), showing an effect restricted to the striolar SCs (Fig. $6 c, d$ ), and similar to P8 utricles cultured in 7F (Fig. 4c). Thus, EC medium is sufficient to induce a decrease in E-cadherin at the junctions of striolar SCs.

Since the treatment with EC medium thinned the F-actin bands and decreased the average intensity of E-cadherin immunostaining in the junctions of striolar SCs, we asked whether it would induce quiescent SCs to enter S-phase. We tested that by culturing P8 utricles in EC medium for $7 \mathrm{~d}$ and adding EdU $48 \mathrm{~h}$ before fixing and immunostaining with antibodies against Sox 2 and oncomodulin. Counts of $\mathrm{EdU}^{+} / \mathrm{Sox}^{+}$cells in the utricles treated with EC medium showed levels of S-phase entry significantly greater than in control cultures and indistinguishable from those in utricles cultured in $7 \mathrm{~F}$ (98 \pm 83 vs $103 \pm 70$, respectively; 7F: $p=$ 0.00529 , two-tailed $t$ test, $n=8$; EC: $p=$ 0.00108 , two-tailed $t$ test, $n=8$ ). Just as in the $7 \mathrm{~F}$-treated utricles, the $\mathrm{EdU}^{+} /$Sox 2

$d$, The $S / L$ ratio of E-cadherin stayed at the same level in P8 mouse utricles cultured in control medium $(n=4$ at each investigated time point for (ontrol and 7F) but progressively decreased in P8 mouse utricles cultured in 7F medium. $\boldsymbol{e}$, $\mathrm{N}$-cadherin $\mathrm{S} / \mathrm{L}$ ratios remained unchanged in both experimental conditions ( $n=4$ at each investigated time point for Control and 7F). Data are mean \pm SD. ns, Not significant ( $p \geq$ $0.05) .{ }^{* *} p<0.001$. DIV, days in vitro. 

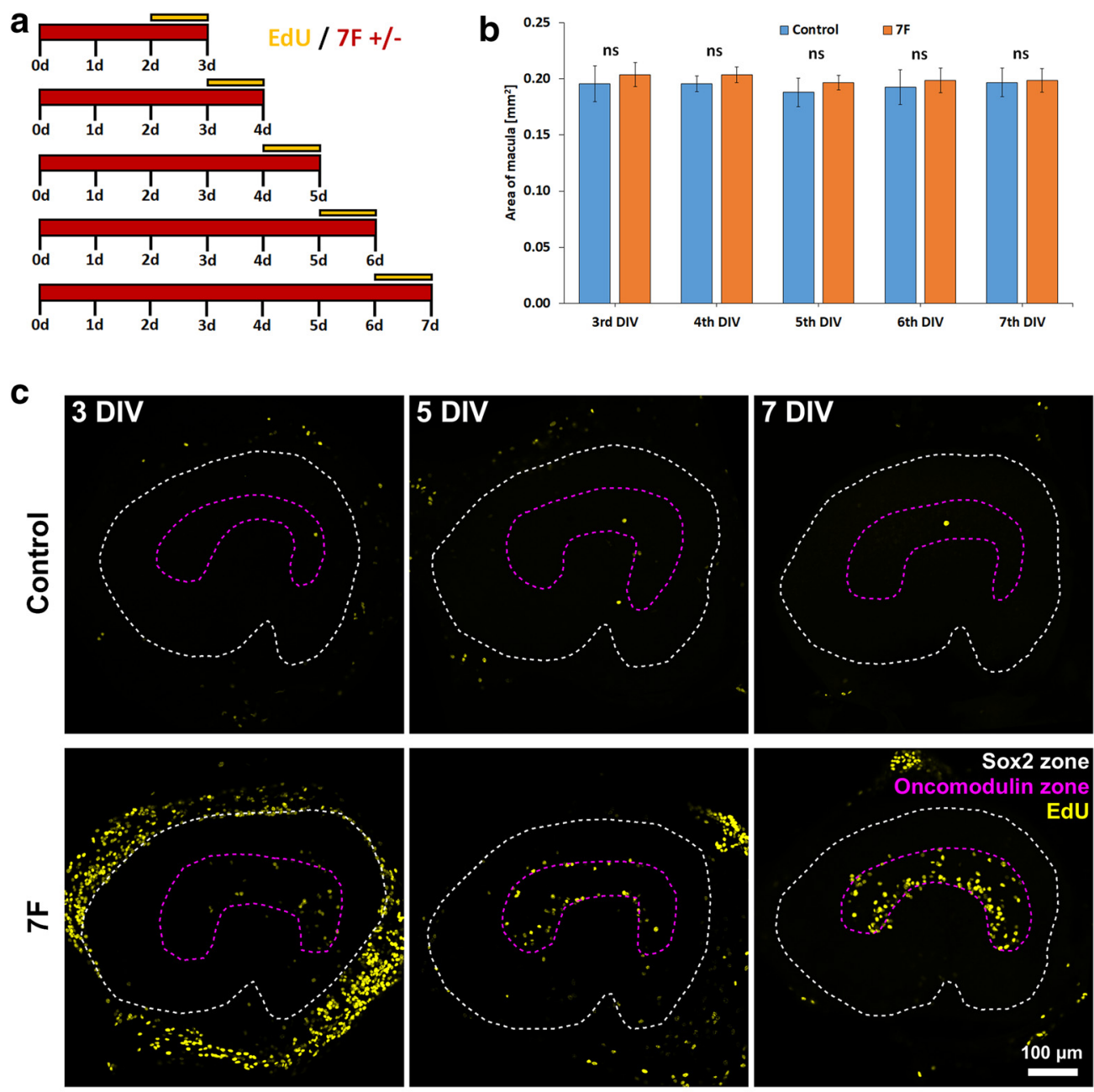

d

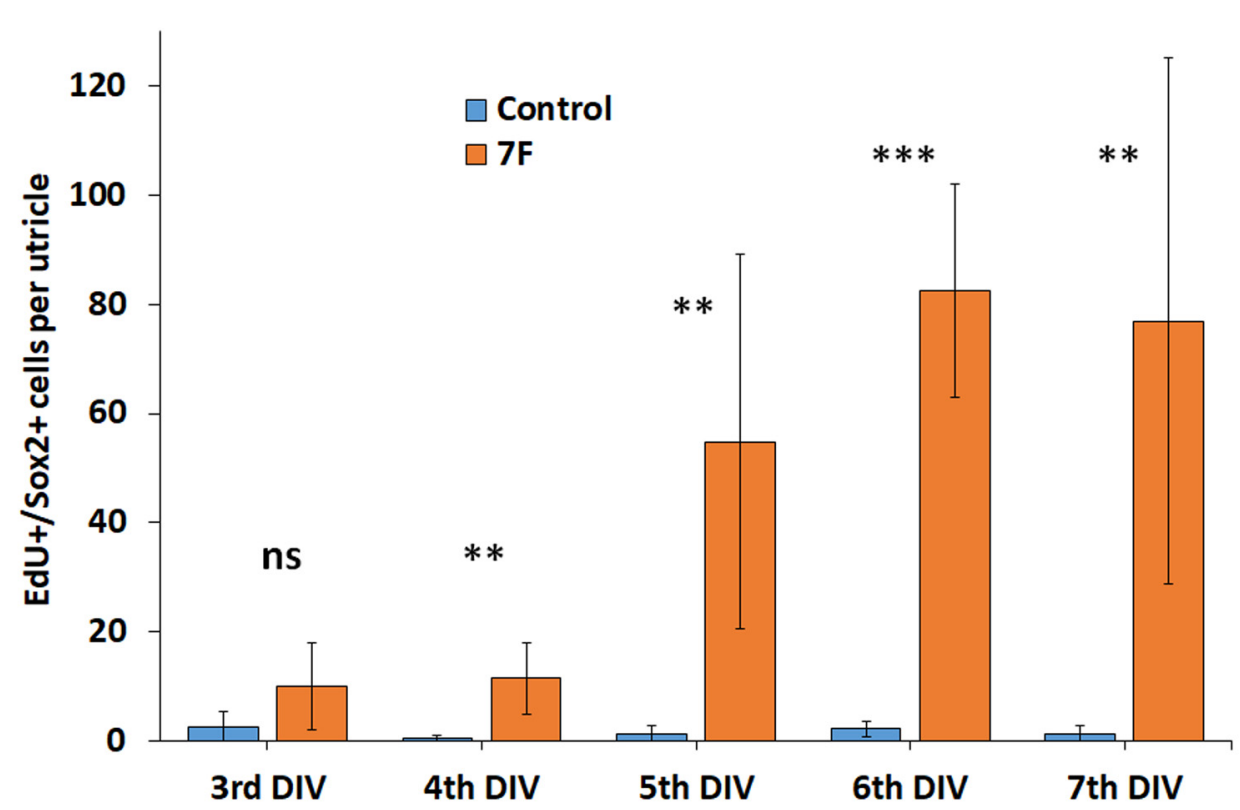

Figure 5. After E-cadherin decreased in the striola of the P8 utricles, many of the normally quiescent SCs in that region reentered the cell cycle and labeled with EdU. $\boldsymbol{a}$, To determine when quiescent SCs began to reenter the cell cycle, utricles from P8 mice were cultured for 3, 4, 5, 6, or $7 \mathrm{~d}$, with EdU added for the final $24 \mathrm{~h}$. $\boldsymbol{b}$, The sensory epithelia of P8 mouse utricles that were cultured in $7 \mathrm{~F} \mathrm{medium} \mathrm{did} \mathrm{not} \mathrm{significantly} \mathrm{expand} \mathrm{relative} \mathrm{to} \mathrm{those} \mathrm{cultured} \mathrm{in} \mathrm{control} \mathrm{medium} \mathrm{(} n=6$ at each investigated time point for Control and $7 \mathrm{~F})$.c, Low-magnification images illustrate the $S$-phase entry of striolar SCs in P8 mouse utricles cultured in the 7F medium. $\boldsymbol{d}$, Quantification showed that the most notable increase in the incidence of S-phase entry in the striolar SCs occurred between the fourth and fifth days of culture ( $n=6$ at each investigated time point for Control and 7F). Data are mean \pm SD. ns, Not significant $(p \geq 0.05) .{ }^{* *} p<0.01,{ }^{* * *} p<0.001$. DIV, days in vitro. 


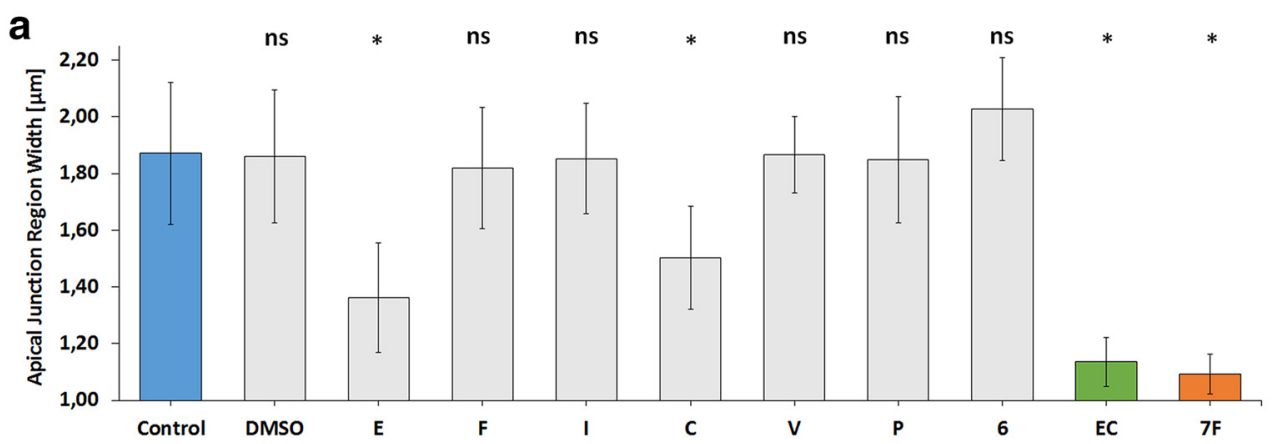

\section{C}

Control
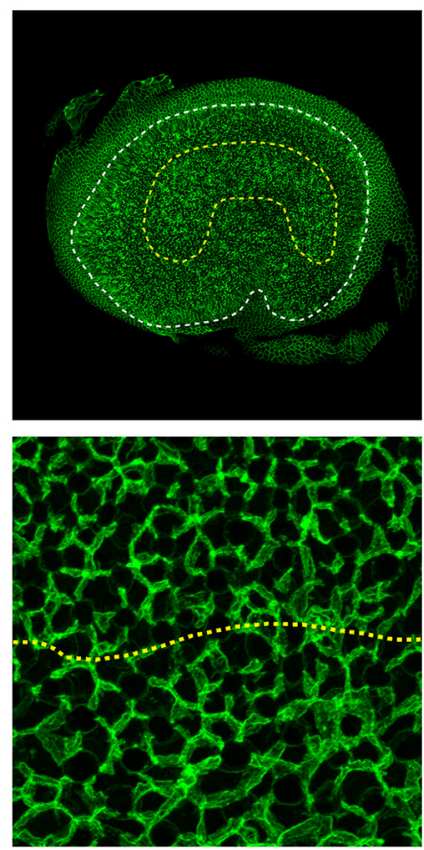

e

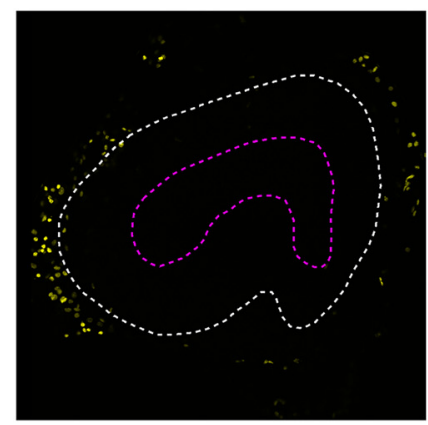

7F
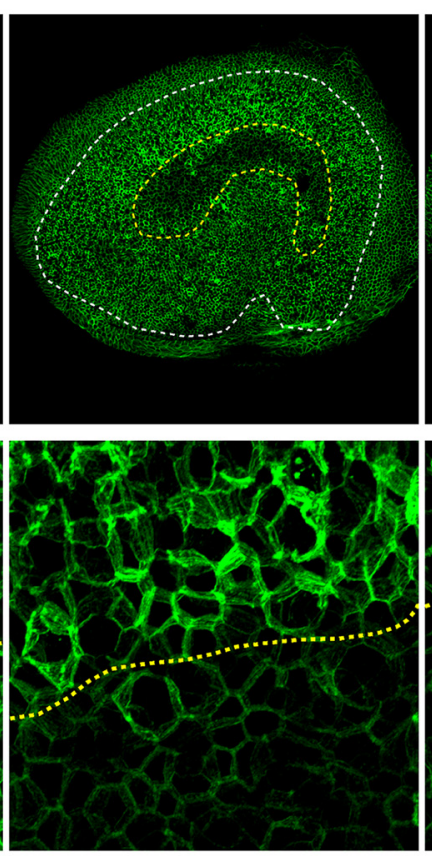

7F

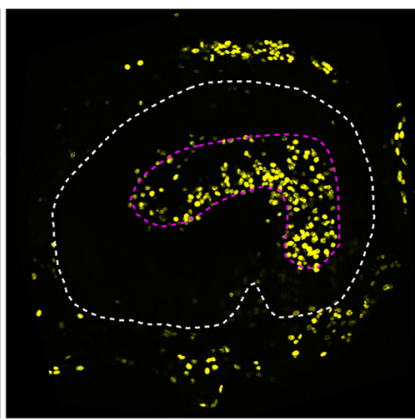

EC
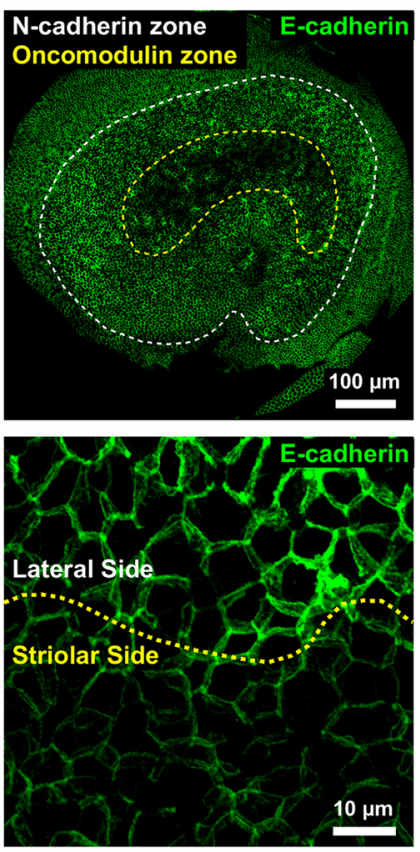

EC

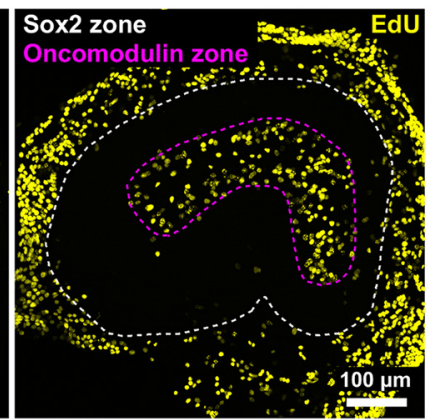

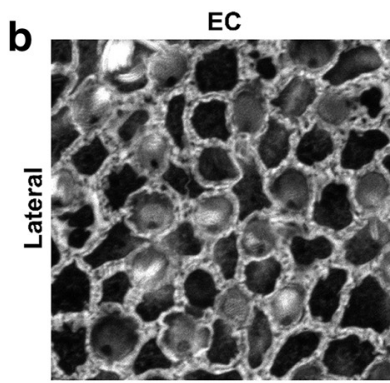
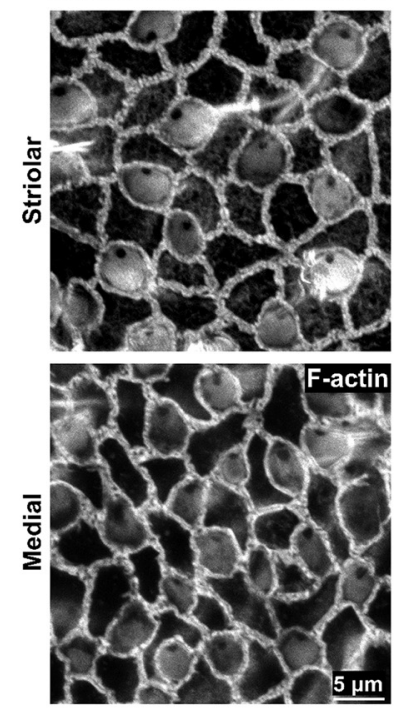

d
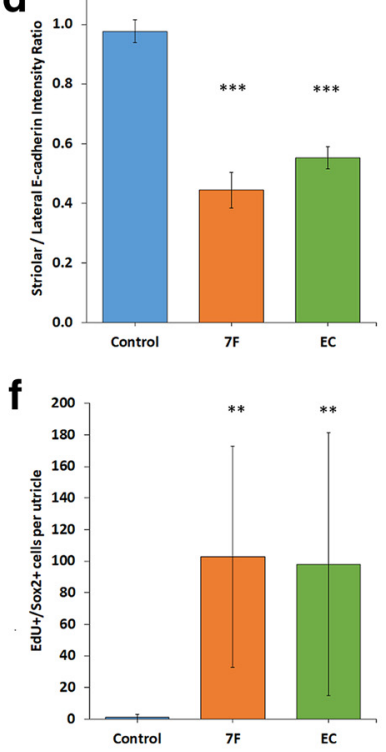

Figure 6. EGF and the GSK3 $\beta$ inhibitor CHIR-99021 are the factors in $7 F$ medium that reverse junctional maturation and induce S-phase entry in striolar SCs. $a, 0 f$ seven factors (E, F, I, C, V, P, 6 ), only EGF (E) and CHIR-99021 (C) significantly decreased the average width of AJRs when individually tested. The effect of E and C resulted in thinning of the AJRs that was comparable with that of $7 F$ medium ( $n=4$ for each experimental condition). Data are mean $\pm S D$. ns, Not significant $(p \geq 0.05) .{ }^{*} p<0.0001$ (ANOVA and then posthoc t test comparing each experimental condition with the control group). $\boldsymbol{b}$, High-magnification images of thinned F-actin bands in P8 mouse utricles cultured in EC medium for $3 \mathrm{~d}$. $\boldsymbol{c}$, Low- and high-magnification images illustrating that levels of $\mathrm{E}$-cadherin immunostaining decreased in striolar SCs of P8 mouse utricles cultured in $\mathrm{EC}$ medium for $5 \mathrm{~d}$. $\boldsymbol{d}$, Culture in $7 \mathrm{~F}$ and $\mathrm{EC}$ medium each significantly decreased the $\mathrm{E}$-cadherin $\mathrm{S} / \mathrm{L}$ ratio compared with control medium ( $n=4$ for Control, $7 \mathrm{~F}$, and EC). Data are mean \pm SD. ns, Not significant ( $p \geq 0.05)$. ${ }^{* * *} p<0.001$ (statistical comparisons are $t$ tests relative to control group).e, Treatment with EC medium was sufficient to induce S-phase entry of striolar SCs in P8 mouse utricles. $f$, Quantification of EdU ${ }^{+} / S 0 \times 2^{+}$cells revealed that culture in EC medium induced a level of $S$-phase entry in SCs comparable with culture in $7 F$ medium ( $n=8$ for Control, 7F, and EC). Data are mean \pm SD. ns, Not significant $(p \geq 0.05)$. ${ }^{* *} p<0.01$ (statistical comparisons are $t$ tests relative to control group).

cells in the EC-cultured utricles were distributed throughout the striola, with a sharp boundary between the zones of EdU ${ }^{+}$striolar SCs and EdU ${ }^{-}$SCs in the surrounding extrastriolar sensory epithelium (Fig. 6e,f).
Treatment with EC medium reduces $\beta$-catenin and YAP in apical domains of striolar SCs

Since the treatment with EGF and CHIR-99021 induced S-phase entry exclusively in the SCs in which junctional E-cadherin had 
a
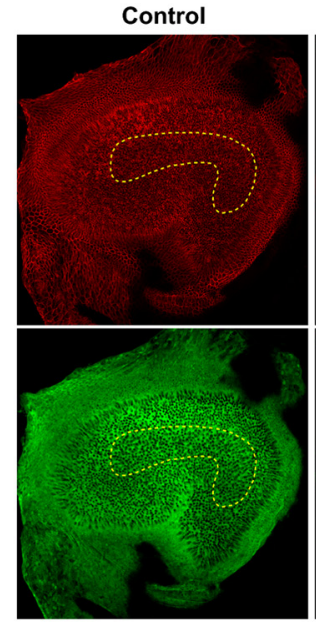

C

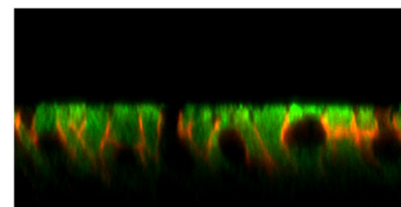

d

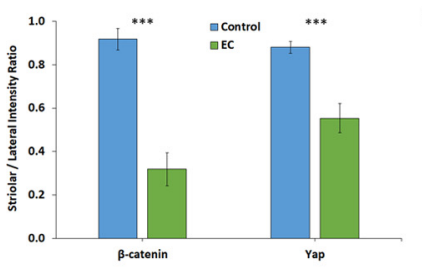

EC

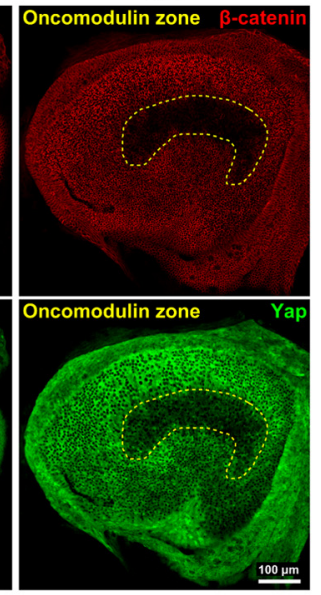

b
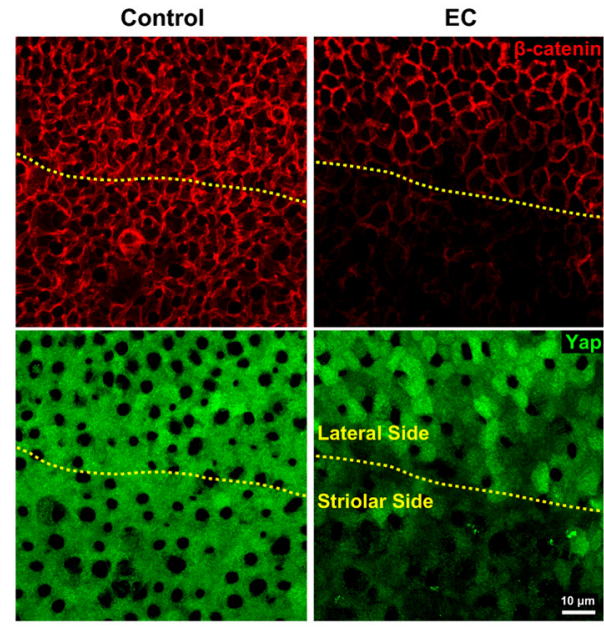

Yap

EdU

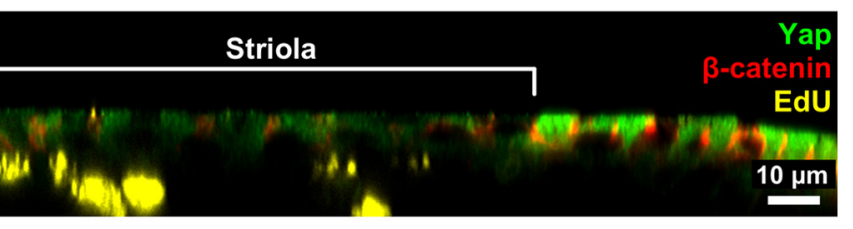

e
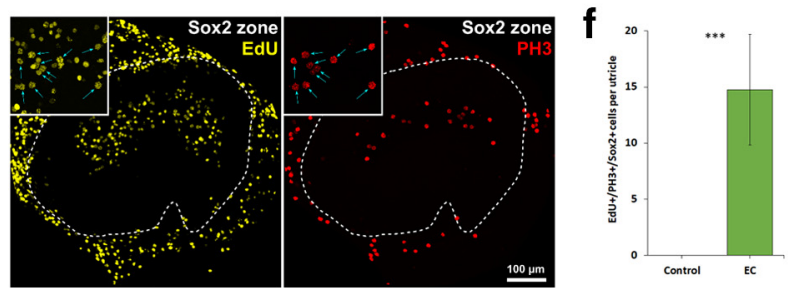

Figure 7. The levels of $\beta$-catenin and YAP decreased in the striola of P8 utricles upon treatment with EGF and the GSK3 inhibitor CHIR-99021. $\boldsymbol{a}$, Low-magnification images illustrating that the intensities of $\beta$-catenin and YAP immunostaining decreased in the striola of P8 mouse utricles cultured in EC medium, which did not occur in control medium. $\boldsymbol{b}$, High-magnification images centered on the line of the polarity reversal (yellow dashes) depicting the decrease in $\beta$-catenin and YAP immunostaining at the cellular level. $c$, Orthogonal view of P8 mouse utricle cultured in EC that illustrates decrease in $\beta$-catenin and YAP immunostaining intensities in the striola and presence of EdU ${ }^{+}$striolar SCs. $\boldsymbol{d}, \beta$-catenin and YAPS/L ratios decreased in P8 mouse utricles cultured in EC medium compared with utricles cultured in control medium ( $n=4$ for Control and EC). $\boldsymbol{e}$, Low-magnification images of P8 mouse utricles cultured in EC medium for $7 \mathrm{~d}$ with EdU and nocodazole added for the final $24 \mathrm{~h}$ of culture. Insets, Cells that progressed from S-phase to mitosis $\left(\mathrm{EdU}{ }^{+} / \mathrm{PH} 3^{+}\right.$cells). $f$, EdU ${ }^{+} / \mathrm{Sox2}{ }^{+} / \mathrm{PH}^{+}{ }^{+}$cells were present only in utricles cultured in $\mathrm{EC}$ medium $(n=$ 4 for Control and EC). Data are mean \pm SD. ns, Not significant $(p \geq 0.05)$. ${ }^{* * *} p<0.001$.

decreased, we decided to investigate the levels of $\beta$-catenin and YAP, junction-associated transcriptional coactivators with proliferative effects. $\beta$-catenin can directly bind to E-cadherin (Nelson and Nusse, 2004), and homophilic interactions between the extracellular domains of E-cadherin at cell junctions are reportedly required for the cytoplasmic sequestration of YAP (Kim et al., 2011; Benham-Pyle et al., 2015). We suspected that the decreases in F-actin and E-cadherin might lead to changes in the amount of $\beta$-catenin or YAP bound at the junctions between striolar SCs, and that such changes could underlie the increase in S-phase entry. To test the first of those hypotheses, we dissected P8 utricles, cultured them in either EC or control medium for $7 \mathrm{~d}$, fixed, and immunostained them with antibodies against $\beta$-catenin, YAP, and oncomodulin.

In the striola of the utricles cultured in EC medium, we observed decreased intensities of $\beta$-catenin and YAP immunostaining relative to the extrastriolar regions of those utricles and all regions of utricles cultured in control medium (Fig. 7a,b). For quantitative comparisons, we captured high-resolution images centered on the line of polarity reversal and calculated S/L ratios for $\beta$-catenin and YAP intensity (see Materials and Methods). In the utricles cultured in control medium, S/L ratios were $0.91 \pm$ 0.05 for $\beta$-catenin and $0.88 \pm 0.03$ for YAP. In the utricles cultured in EC medium, the S/L ratio for $\beta$-catenin decreased to
$0.32 \pm 0.08(p=0.00001$, two-tailed $t$ test, $n=4)$ and the S/L ratio for YAP decreased to $0.55 \pm 0.07$ ( $p=0.00010$, two-tailed $t$ test, $n=4$ ) (Fig. $7 d$ ). The striolar-specific decreases in Ecadherin, $\beta$-catenin, and YAP are consistent with the hypothesis that those transcriptional coactivators participate in the transition of maturing SCs to cells that enter S-phase.

To investigate whether the maturing striolar SCs that entered S-phase would progress to mitosis, we cultured P8 utricles in either EC medium or control medium for $7 \mathrm{~d}$. For the last day of culture, we added EdU to label cells that entered S-phase and nocodazole to arrest cells in mitosis. Then we immunostained for the mitosis marker phosphorylated histone-3 (PH3).

We observed robust S-phase entry in striolar SCs of the P8 utricles cultured in EC medium $\left(154 \pm 54 \mathrm{EdU}^{+} / \mathrm{Sox} 2^{+}\right.$cells $)$, $15 \pm 5$ cells of which were $\mathrm{EdU}^{+} / \mathrm{Sox} 2^{+} / \mathrm{PH} 3^{+}$, demonstrating that maturing SCs in P8 utricles are able to progress to mitosis (Fig. $7 e, f$ ). As in earlier experiments, $\mathrm{EdU}^{+} / \mathrm{Sox} 2^{+}$cells were rarely found in the P8 utricles cultured in control medium, and none was positive for $\mathrm{PH} 3$.

EC medium reverses junctional maturation and promotes proliferation of striolar SCs in utricles from adult mice Since the combination of EGF and CHIR-99021 thinned the F-actin bands throughout P8 utricles and led to reductions in 
E-cadherin, $\beta$-catenin, and YAP, inducing SCs in the striola to enter S-phase and progress in the cell cycle, we sought to determine whether EC medium would cause the long-quiescent SCs in adult utricles to enter S-phase. Earlier studies showed that the junctional F-actin bands in adult utricles are so thick that they occupy $89 \%$ of cell area at the level of the intercellular junction in the average SC (Burns et al., 2008). The junctions of adult SCs in both the utricle and the cochlea also contain correspondingly high levels of E-cadherin (Collado et al., 2011b; Burns et al., 2013). Therefore, we extended the duration of the culture period from $3 \mathrm{~d}$ (Fig. 1) to $11 \mathrm{~d}$ to allow more time for the effects of EC medium to manifest in adult SCs.

As in the utricles from younger mice, culture of adult utricles in EC medium resulted in significantly thinner AJRs than culture in control medium $(1.29 \pm 0.03 \mathrm{vs}$ $3.06 \pm 1.08 \mu \mathrm{m} ; p<0.00001$, two-tailed $t$ test, $n=4$ ) (Fig. $8 a, b$ ). Likewise, measurements of the intensities of E-cadherin, $\mathrm{N}$-cadherin, $\beta$-catenin, and YAP immunostaining in adult utricles cultured in EC medium revealed significantly lower S/L ratios for E-cadherin ( $p=0.00552$, twotailed $t$ test, $n=4), \beta$-catenin ( $p=$ 0.00029 , two-tailed $t$ test, $n=4)$, and YAP ( $p=0.00279$, two-tailed $t$ test, $n=4)$, but not $\mathrm{N}$-cadherin $(p=0.55563$, two-tailed $t$ test, $n=4)$, when comparisons were made to adult utricles cultured in control medium (Fig. 9a-c).

Finally, to determine whether it would be possible to stimulate fully mature SCs to proliferate, we cultured utricles from adult mice in EC medium and added EdU for the final $48 \mathrm{~h}$ before fixation. The adult utricles cultured in EC medium contained on average $104 \pm 40 \mathrm{EdU}^{+} /$Sox $2^{+}$cells, 50fold more than the utricles cultured in control medium $(2 \pm 2$ $\mathrm{EdU}^{+} /$Sox $2^{+} ; p=0.00002$, two-tailed $t$ test, $n=7$ ) (Fig. 9d,e), and nearly all of the EdU ${ }^{+} /$Sox $2^{+}$cells were in the striola (Fig. $9 e$ ). In a modification of the experiment, we added EdU and nocodazole $24 \mathrm{~h}$ before fixation to test whether fully mature and normally quiescent striolar SCs were blocked from cell cycle progression. On average, $3 \pm 2 \mathrm{PH}^{+} / \mathrm{EdU}^{+} / \mathrm{Sox} 2{ }^{+}$cells were observed in the utricles treated with EC medium (Fig. $8 \mathrm{c}$ ), and none were found in the utricles cultured in control medium.

Together, the findings indicate that stimulation with EGF and an inhibitor of GSK3 promotes proliferation of mature SCs in the striola of adult mice, which correlates in timing and location with thinning of the uniquely massive F-actin bands and decreases in the levels of E-cadherin, $\beta$-catenin, and YAP at the apical domains and cell junctions.

\section{Discussion}

Vertebrate SCs share most characteristics, but vestibular SCs in mammals are distinct from those in nonmammals in that their intercellular junctions develop high levels of E-cadherin and unique, stable, and exceptionally thick circumferential F-actin bands, which grow to occupy $89 \%$ of the adult SC's area at the junction level (Meyers and Corwin, 2007; Burns et al., 2008; Collado et al., 2011b; Burns and Corwin, 2014). Neither occurs in the SCs of fish, amphibians, and birds that readily regenerate HCs and recover sensory function (Burns et al., 2013). Those discoveries led to the hypothesis that mammalian SC junctions play a role in limiting proliferation. Our findings here have shown that exposure to EGF and CHIR-99021 caused SC junctions in utricles from mature mice to revert to thin circumferential F-actin bands like those found in the SCs of nonmammals and embryonic mammals, with thinning occurring throughout the sensory epithelium. When treatment duration was extended, junctional E-cadherin decreased significantly, but only in SCs within the striola. That, in turn, was followed by a dramatic restoration of the capacity for the adult striolar SCs to proliferate, consistent with expectations based on the hypothesis.

Prior experiments showed that F-actin near the junction is sensitive to latrunculin A, resistant to cytochalasin D, and relatively mobile, whereas $\mathrm{F}$-actin in a zone further from the junction is resistant to latrunculin A, sensitive to cytochalasin D, and relatively immobile (Burns and Corwin, 2014). It appears that EGF and CHIR-99021 act on the latter zone, causing AJR widths to thin until they stabilize at $\sim 1.0 \mu \mathrm{m}$. While thinning of the circumferential bands may play a role in returning mammalian SCs to a proliferative state, it was not sufficient to release them from quiescence. Thinning occurred throughout the utricular SC population, but proliferation occurred only in striolar SCs where 
a
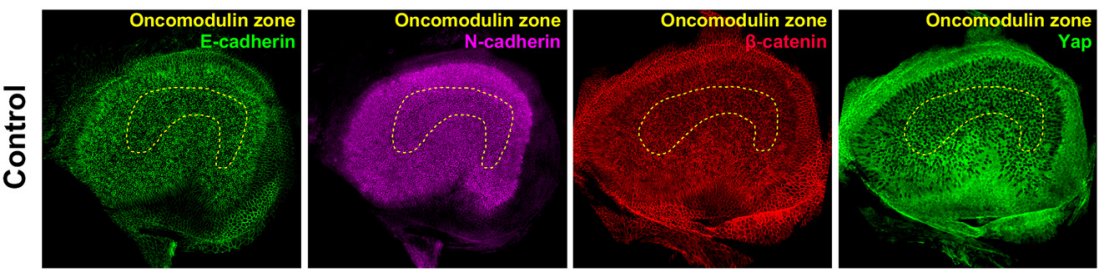

U
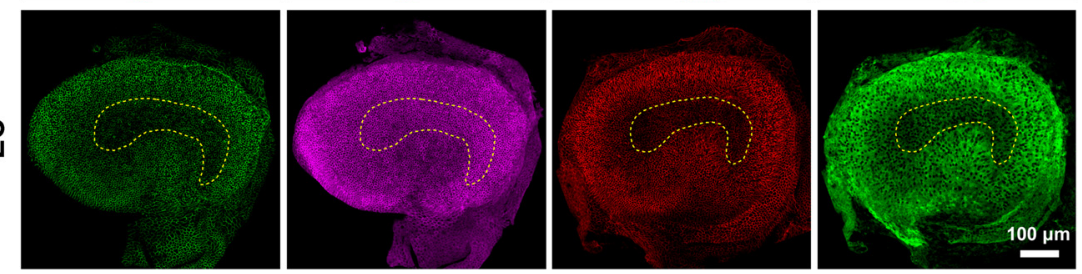

b
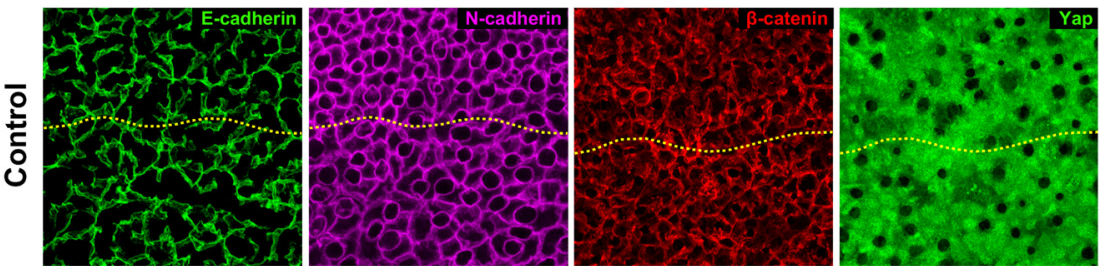

U
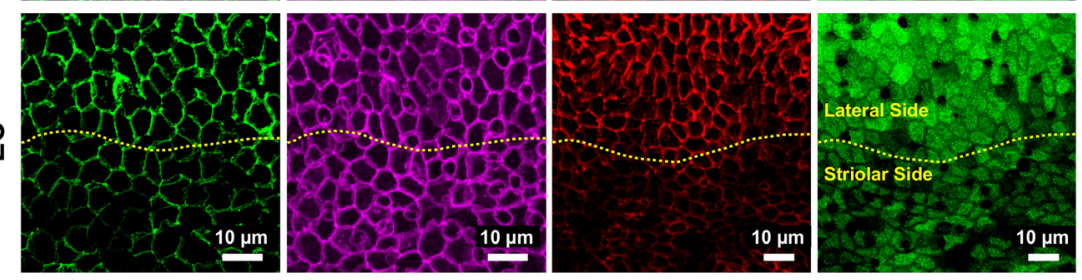

C

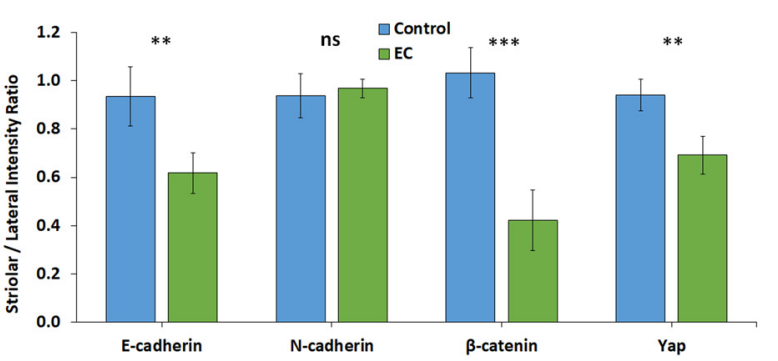

d

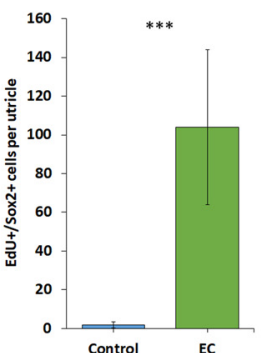

e

Control

EC
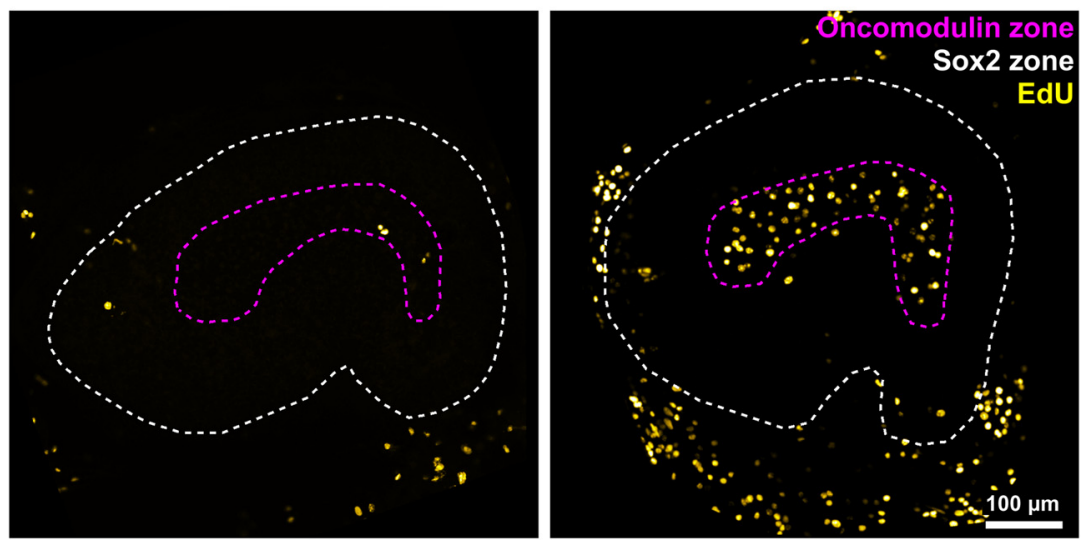

Figure 9. EGF and the GSK3 $\beta$ inhibitor CHIR-99021 reverse the reinforcement of SC-SC junctions and promote proliferation of striolar SCs in utricles from adult mice. $\boldsymbol{a}$, Low-magnification images illustrate that culture in $\mathrm{EC}$ medium for $11 \mathrm{~d}$ decreases the intensities of E-cadherin, $\beta$-catenin, and YAP immunostaining, but not $\mathrm{N}$-cadherin immunostaining, in the striola of adult mouse utricles. Such decreases did not occur in the control medium. $\boldsymbol{b}$, High-magnification images centered on the line of the polarity reversal (yellow dashes) depicting E-cadherin, $\mathrm{N}$-cadherin $\beta$-catenin, and YAP immunostaining at the cellular level. $c$, The $\mathrm{S} / \mathrm{L}$ ratios of $\mathrm{E}$-cadherin, $\beta$-catenin, and YAP significantly decreased in adult mouse utricles cultured in $\mathrm{EC}$ medium compared with utricles cultured in control medium ( $n=4$ for Control and EC). The S/L ratio of N-cadherin did not differ significantly. $d$, Quantification of EdU ${ }^{+} / S_{0 \times 2}{ }^{+}$cells revealed that culture in $\mathrm{EC}$ medium induced a 50 -fold increase in the number of SCs that entered S-phase compared with the number of such SCs found in utricles cultured in control medium ( $n=7$ for Control and EC).e, As in P8 utricles, culture of adult mouse utricles in EC medium induced S-phase entry in striolar SCs. Data are mean \pm SD. ns, Not significant $(p \geq 0.05)$. ${ }^{* *} p<0.01,{ }^{* * *} p<0.001$. 
junctional E-cadherin decreased. Since downregulation of E-cadherin occurred just before the SCs' transition from quiescence to proliferation, it appears likely that the high levels of junctional E-cadherin are responsible for the quiescence of mature mammalian SCs. In cancer, downregulation of E-cadherin is strongly correlated with invasiveness, progression to malignancy, and poorer prognosis, hence its designation as a tumor suppressor gene (Pećina-Slaus, 2003; Q. P. Hu et al., 2016).

In an earlier attempt to investigate the role of E-cadherin in mammalian SCs, we generated conditional genetic KOs and found that many weeks were required before E-cadherin depletion became evident at utricular SC junctions (M. S. Collado and J.T.C., unpublished results). In sharp contrast to that, the treatment with 7F led to a significant decrease in the expression of junctional E-cadherin in just $3 \mathrm{~d}$ (Fig. 4), raising the possibility that its thinning of the circumferential F-actin bands accelerated the rate of E-cadherin depletion. Indeed, F-actin has been shown to stabilize E-cadherin clusters in cultured epithelial cells (Hong et al., 2013).

Our findings are consistent with the possibility that the release of apically localized $\beta$-catenin and YAP promotes cell cycle entry downstream of junctional E-cadherin depletion. E-cadherin adhesions are known to bind $\beta$-catenin directly and contribute to the cytoplasmic sequestration of YAP, limiting the ability for those transcriptional coactivators to induce proliferation in cultured cell lines (Heuberger and Birchmeier, 2010; Kim et al., 2011; Benham-Pyle et al., 2015). In utricles from neonatal mice, the canonical Wnt and YAP-TEAD pathways have been shown to mediate SC proliferation (Wang et al., 2015; L. Hu et al., 2016; Gnedeva et al., 2017). Our results in the striola build on that and suggest that E-cadherin mediated inhibition of the Wnt and YAPTEAD pathways plays an important role in keeping mature vestibular SCs of mammals in a quiescent state.

Ligands such as EGF, TGF- $\alpha$, and neuregulins have been shown to promote proliferation of SCs in cultured mammalian balance epithelia (Lambert, 1994; Yamashita and Oesterle, 1995; Zheng et al., 1997; Kuntz and Oesterle, 1998; Montcouquiol and Corwin, 2001). EGFR signaling also stimulates regenerative proliferation of SCs in the cochlear epithelia of birds and has stimulatory effects on a subset of SCs after dissociation from the cochlea of neonatal mammals (White et al., 2012). Indeed, evidence in that study indicates that EGFR signaling can lift the $\mathrm{p} 27^{\mathrm{kip} 1}$ inhibition of proliferation in the dissociated SCs, and suggests that a PI3K-dependent pathway appears to play a role in the $\mathrm{p} 27^{\mathrm{kip} 1}$-independent proliferation of that subset of dissociated SCs from the neonatal mouse cochlea. The mitogenic effect of GSK3 inhibitors in neonatal HC epithelia is also well documented (Lu and Corwin, 2008; Roccio et al., 2015; Samarajeewa et al., 2018; You et al., 2018). Our results suggest that the stimulatory effects of these agents are closely linked with their capacity to remodel junctions once SCs have matured. However, questions remain about how EGF and the GSK3 inhibitor brought about the depletion of E-cadherin in the striolar SCs. Since the level of junctional $\mathrm{N}$-cadherin remained unchanged, we can conclude that the mechanism of depletion is selective for E-cadherin, and does not act as a "cadherin switch" where downregulation of E-cadherin is accompanied by upregulation of $\mathrm{N}$-cadherin as in epithelial-mesenchymal transition (Figs. 4, 8) (Wheelock et al., 2008). Inhibition of GSK3 $\beta$ has been shown to increase transcription of Snail, which deacetylates the $C d h 1$ promoter, so the CHIR-99021-mediated depletion of E-cadherin could come about through a decrease in its transcription (Batlle et al., 2000; Bachelder et al., 2005; Lu and Corwin, 2008; Collado et al., 2011b;
Kim et al., 2012; Liu et al., 2014). Indeed, EGFR activation inhibits GSK3 activity and leads to epithelial-mesenchymal transition. EGF also has been shown to induce E-cadherin internalization via caveolin-mediated endocytosis (Lu et al., 2003), micropinocytosis (Bryant et al., 2007), and following Hakai-mediated ubiquitination via Src (Shen et al., 2008). EGF also can also induce proteolytic cleavage of E-cadherin by matrix metalloproteases (Cowden Dahl et al., 2008; Zheng et al., 2009; Chavez et al., 2012; Navarini et al., 2017). Additional studies will be required to determine which of those potential mechanisms may be responsible for the depletion of E-cadherin that occurs in striolar SCs exposed to EGF and CHIR-99021.

The distinct effects we observed in striolar and extrastriolar SCs indicate that yet-to-be-identified properties of extrastriolar SCs prevent them from downregulating E-cadherin and proliferating in response to EGF signaling and GSK3 inhibition. Since incidental HC loss occurs in both striolar and extrastriolar regions, stiolar-specific losses do not appear likely to account for the region-specific effects observed. Previous studies have reported the following differences between striolar and extrastriolar SCs: Striolar SCs express the stem cell marker Lgr5 at embryonic stages, are more likely to proliferate after diphtheriatoxin-mediated $\mathrm{HC}$ ablation at $\mathrm{P} 1$, and are more likely to convert into HCs upon Notch inhibition before P12 than extrastriolar SCs (Collado et al., 2011b; Wang et al., 2015). In contrast, the limited nonproliferative $\mathrm{HC}$ replacement that occurs in adult mouse utricles after diphtheria-toxin-mediated HC ablation primarily occurs in the extrastriolar region, via direct phenotypic conversion (Golub et al., 2012). The identification of differences between striolar and extrastriolar SCs appears likely to reveal important clues about barriers that limit HC replacement in mammals. Recent research has shown that the developmental specification of striolar and extrastriolar regions is controlled by regional differences in retinoic acid metabolism, with higher retinoic acid levels in the extrastriolar epithelium (Ono et al., 2020). This raises the possibility that retinoic acid signaling, which is known to have diverse effects, including the promotion of cell cycle exit and differentiation in neural progenitors (Janesick et al., 2015), may lead to gene expression changes that limit the plasticity of extrastriolar SCs.

Proliferation in the adult mammalian utricle is extremely limited during homeostasis (Bucks et al., 2017), and even after extensive HC loss (Warchol et al., 1993; Golub et al., 2012; Taylor et al., 2018). Replacement HCs can arise as SCs convert or directly transdifferentiate into HCs without an intervening cell division. That process appears to restore a significant, but limited fraction of the HCs and depletes the SC population (Golub et al., 2012; Taylor et al., 2018). In nonmammalian vertebrates, SC proliferation is often the primary contributor to the regenerative replacement of lost HCs (Corwin and Cotanche, 1988; Ryals and Rubel, 1988; Stone and Rubel, 1999; Wibowo et al., 2011; Mackenzie and Raible, 2012) and also serves to replenish SC numbers following direct transdifferentiation (Scheibinger et al., 2018). Adult murine SCs will enter the cell cycle after dramatic shape changes (Meyers and Corwin, 2007), after delaminaton when cultured on rigid substrates (Collado et al., 2011a), when transduced with oncogenes (Loponen et al., 2011; Burns et al., 2012b), or when dissociated from the epithelium (Li et al., 2003; Oshima et al., 2007; McLean et al., 2017). Here we report that adult mammalian SCs in undamaged vestibular epithelia proliferate robustly after a relatively simple 11-d-long pharmacological treatment, which led to proliferation of $104 \pm 40$ SCs during the final $48 \mathrm{~h}$ of the treatment (Fig. 9d,e). The results illustrate the potential for phar- 
macological approaches to promote repair in the mammalian ear.

In conclusion, findings outlined above support the hypothesis that the reinforcement of intercellular junctions, which takes place in mammalian ears but not in fish, amphibians, or birds, plays an important role in preventing mammalian SCs from proliferating. Our findings demonstrate, for the first time, that the partial dismantling of those junctions precedes and correlates with a resurgence of proliferation in the long-quiescent SCs of mature mammalian ears. While strengthening support for the hypothesis, these findings do not establish causality, since the reversion to less specialized junctional characteristics and the transition from quiescence to proliferation could occur independently in response to a common upstream control. Nonetheless, our results suggest that high expression of E-cadherin regulates signaling that maintains SCs in a quiescent state that appears to be responsible, in part, for the mammalian ear's vulnerability to permanent balance and hearing deficits.

\section{References}

Agrawal Y, Carey JP, Della Santina CC, Schubert MC, Minor LB (2009) Disorders of balance and vestibular function in US adults: data from the National Health and Nutrition Examination Survey, 2001-2004. Arch Intern Med 169:938-944.

Bachelder RE, Yoon SO, Franci C, de Herreros AG, Mercurio AM (2005) Glycogen synthase kinase- 3 is an endogenous inhibitor of snail transcription. J Cell Biol 168:29-33.

Batlle E, Sancho E, Francí C, Domínguez D, Monfar M, Baulida J, García DeHerreros AG de (2000) The transcription factor Snail is a repressor of E-cadherin gene expression in epithelial tumour cells. Nat Cell Biol 2:8489.

Benham-Pyle BW, Pruitt BL, Nelson WJ (2015) Mechanical strain induces E-cadherin-dependent Yap1 and $\beta$-catenin activation to drive cell cycle entry. Science 348:1024-1027.

Bryant DM, Kerr MC, Hammond LA, Joseph SR, Mostov KE, Teasdale RD, Stow JL (2007) EGF induces macropinocytosis and SNX1-modulated recycling of E-cadherin. J Cell Sci 120:1818-1828.

Bucks SA, Cox BC, Vlosich BA, Manning JP, Nguyen TB, Stone JS (2017) Supporting cells remove and replace sensory receptor hair cells in a balance organ of adult mice. eLife 6:e18128.

Burns JC, Corwin JT (2013) A historical to present-day account of efforts to answer the question, "What puts the brakes on mammalian hair cell regeneration?" Hear Res 297:52-67.

Burns JC, Corwin JT (2014) Responses to cell loss become restricted as the supporting cells in mammalian vestibular organs grow thick junctional actin bands that develop high stability. J Neurosci 34:1998-2011.

Burns JC, Christophel JJ, Collado MS, Magnus C, Carfrae M, Corwin JT (2008) Reinforcement of cell junctions correlates with the absence of hair cell regeneration in mammals and its occurrence in birds. J Comp Neurol 511:396-414.

Burns JC, Cox BC, Thiede BR, Zuo J, Corwin JT (2012a) In vivo proliferative regeneration of balance hair cells in newborn mice. J Neurosci 32: $6570-6577$.

Burns JC, Yoo JJ, Atala A, Jackson JD (2012b) MYC gene delivery to adult mouse utricles stimulates proliferation of postmitotic supporting cells in vitro. PLoS One 7:e48704.

Burns JC, Collado MS, Oliver ER, Corwin JT (2013) Specializations of intercellular junctions are associated with the presence and absence of hair cell regeneration in ears from six vertebrate classes. J Comp Neurol 521: $1430-1448$.

Chavez MG, Buhr CA, Petrie WK, Wandinger-Ness A, Kusewitt DF, Hudson LG (2012) Differential Downregulation of E-Cadherin and Desmoglein by Epidermal Growth Factor. Dermatology Research and Practice Available at: https://www.hindawi.com/journals/drp/2012/309587/. Accessed September 13, 2019.

Collado MS, Burns JC, Meyers JR, Corwin JT (2011a) Variations in shapesensitive restriction points mirror differences in the regeneration capacities of avian and mammalian ears. PLoS One 6:e23861.

Collado MS, Thiede BR, Baker W, Askew C, Igbani LM, Corwin JT (2011b) The postnatal accumulation of junctional E-cadherin is inversely corre- lated with the capacity for supporting cells to convert directly into sensory hair cells in mammalian balance organs. J Neurosci 31:11855-11866.

Corwin JT, Cotanche DA (1988) Regeneration of sensory hair cells after acoustic trauma. Science 240:1772-1774.

Cowden Dahl KD, Symowicz J, Ning Y, Gutierrez E, Fishman DA, Adley BP, Stack MS, Hudson LG (2008) Matrix metalloproteinase (MMP)-9 is a mediator of epidermal growth factor dependent E-cadherin loss in ovarian carcinoma cells. Cancer Res 68:4606-4613.

Davies D, Magnus C, Corwin JT (2007) Developmental changes in cellextracellular matrix interactions limit proliferation in the mammalian inner ear. Eur J Neurosci 25:985-998.

Gnedeva K, Jacobo A, Salvi JD, Petelski AA, Hudspeth AJ (2017) Elastic force restricts growth of the murine utricle. eLife 6:e25681.

Golub JS, Tong L, Ngyuen TB, Hume CR, Palmiter RD, Rubel EW, Stone JS (2012) Hair cell replacement in adult mouse utricles after targeted ablation of hair cells with diphtheria toxin. J Neurosci 32:15093-15105.

Gu R, Montcouquiol M, Marchionni M, Corwin JT (2007) Proliferative responses to growth factors decline rapidly during postnatal maturation of mammalian hair cell epithelia. Eur J Neurosci 25:1363-1372.

Hackett L, Davies D, Helyer R, Kennedy H, Kros C, Lawlor P, Rivolta MN, Holley M (2002) E-cadherin and the differentiation of mammalian vestibular hair cells. Exp Cell Res 278:19-30.

Heuberger J, Birchmeier W (2010) Interplay of cadherin-mediated cell adhesion and canonical Wnt signaling. Cold Spring Harb Perspect Biol 2:a002915.

Hong S, Troyanovsky RB, Troyanovsky SM (2013) Binding to F-actin guides cadherin cluster assembly, stability, and movement. J Cell Biol 201:131-143.

Hu L, Lu J, Chiang H, Wu H, Edge AS, Shi F (2016) Diphtheria toxininduced cell death triggers wnt-dependent hair cell regeneration in neonatal mice. J Neurosci 36:9479-9489.

Hu QP, Kuang JY, Yang QK, Bian XW, Yu SC (2016) Beyond a tumor suppressor: soluble E-cadherin promotes the progression of cancer. Int J Cancer 138:2804-2812.

Hume CR, Kirkegaard M, Oesterle EC (2003) ErbB expression: the mouse inner ear and maturation of the mitogenic response to heregulin. J Assoc Res Otolaryngol 4:422-443.

Janesick A, Wu SC, Blumberg B (2015) Retinoic acid signaling and neuronal differentiation. Cell Mol Life Sci 72:1559-1576.

Jones JE, Corwin JT (1996) Regeneration of sensory cells after laser ablation in the lateral line system: hair cell lineage and macrophage behavior revealed by time-lapse video microscopy. J Neurosci 16:649-662.

Kim JY, Kim YM, Yang CH, Cho SK, Lee JW, Cho M (2012) Functional regulation of slug/Snail2 is dependent on GSK-3 $\beta$-mediated phosphorylation. FEBS J 279:2929-2939.

Kim NG, Koh E, Chen X, Gumbiner BM (2011) E-cadherin mediates contact inhibition of proliferation through hippo signaling-pathway components. Proc Natl Acad Sci U S A 108:11930-11935.

Kuntz AL, Oesterle EC (1998) Transforming growth factor $\alpha$ with insulin stimulates cell proliferation in vivo in adult rat vestibular sensory epithelium. J Comp Neurol 399:413-423.

Lambert PR (1994) Inner ear hair cell regeneration in a mammal: identification of a triggering factor. Laryngoscope 104:701-718.

Li A, Xue J, Peterson EJ (2008) Architecture of the mouse utricle: macular organization and hair bundle heights. J Neurophysiol 99:718-733.

Li H, Liu H, Heller S (2003) Pluripotent stem cells from the adult mouse inner ear. Nat Med 9:1293-1299.

Liu ZC, Chen XH, Song HX, Wang HS, Zhang G, Wang H, Chen DY, Fang R, Liu H, Cai SH, Du J (2014) Snail regulated by PKC/GSK- $3 \beta$ pathway is crucial for EGF-induced epithelial-mesenchymal transition (EMT) of cancer cells. Cell Tissue Res 358:491-502.

Loponen H, Ylikoski J, Albrecht JH, Pirvola U (2011) Restrictions in cell cycle progression of adult vestibular supporting cells in response to ectopic cyclin D1 expression. PLoS One 6:e27360.

Lu Z, Corwin JT (2008) The influence of glycogen synthase kinase 3 in limiting cell addition in the mammalian ear. Dev Neurobiol 68:1059-1075.

Lu Z, Ghosh S, Wang Z, Hunter T (2003) Downregulation of caveolin-1 function by EGF leads to the loss of E-cadherin, increased transcriptional activity of beta-catenin, and enhanced tumor cell invasion. Cancer Cell 4:499-515.

Mackenzie SM, Raible DW (2012) Proliferative regeneration of zebrafish lateral line hair cells after different ototoxic insults. PLoS One 7:e47257. 
McLean WJ, Yin X, Lu L, Lenz DR, McLean D, Langer R, Karp JM, Edge AS (2017) Clonal expansion of Lgr5-positive cells from mammalian cochlea and high-purity generation of sensory hair cells. Cell Rep 18:1917-1929.

Meyers JR, Corwin JT (2007) Shape change controls supporting cell proliferation in lesioned mammalian balance epithelium. J Neurosci 27: 4313-4325.

Montcouquiol M, Corwin JT (2001) Intracellular signals that control cell proliferation in mammalian balance epithelia: key roles for phosphatidylinositol-3 kinase, mammalian target of rapamycin, and S6 kinases in preference to calcium, protein kinase $\mathrm{C}$, and mitogen-activated protein kinase. J Neurosci 21:570-580.

Nadol JB Jr (1993) Hearing loss. N Engl J Med 329:1092-1102.

Navarini NF, De Araújo VC, Sperandio M, Napimoga MH, Teixeira LN, De Araújo NS, Martinez EF (2017) Effect of epithelial growth factor on matrix metalloproteinase- 2 and $\mathrm{E}$-cadherin $/ \beta$-catenin expression in an in situ model of tumorigenesis. Oncol Lett 14:3136-3140.

Nelson WJ, Nusse R (2004) Convergence of wnt, $\beta$-catenin, and cadherin pathways. Science 303:1483-1487.

Ono K, Keller J, López Ramírez O, González Garrido A, Zobeiri OA, Chang HH, Vijayakumar S, Ayiotis A, Duester G, Della Santina CC, Jones SM, Cullen KE, Eatock RA, Wu DK (2020) Retinoic acid degradation shapes zonal development of vestibular organs and sensitivity to transient linear accelerations. Nat Commun 11:63.

Oshima K, Grimm CM, Corrales CE, Senn P, Martinez Monedero R, Géléoc GS, Edge A, Holt JR, Heller S (2007) Differential distribution of stem cells in the auditory and vestibular organs of the inner ear. J Assoc Res Laryngol 8:18-31.

Pećina-Slaus N (2003) Tumor suppressor gene E-cadherin and its role in normal and malignant cells. Cancer Cell Int 3:17.

Roccio M, Hahnewald S, Perny M, Senn P (2015) Cell cycle reactivation of cochlear progenitor cells in neonatal FUCCI mice by a GSK3 small molecule inhibitor. Sci Rep 5:17886.

Ryals BM, Rubel EW (1988) Hair cell regeneration after acoustic trauma in adult coturnix quail. Science 240:1774-1776.

Samarajeewa A, Lenz DR, Xie L, Chiang H, Kirchner R, Mulvaney JF, Edge AS, Dabdoub A (2018) Transcriptional response to Wnt activation regulates the regenerative capacity of the mammalian cochlea. Development 145:dev166579.

Scheibinger M, Ellwanger DC, Corrales CE, Stone JS, Heller S (2018) Aminoglycoside damage and hair cell regeneration in the chicken utricle. J Assoc Res Laryngol 19:17-29.

Shen Y, Hirsch DS, Sasiela CA, Wu WJ (2008) Cdc42 regulates E-cadherin ubiquitination and degradation through an epidermal growth factor receptor to src-mediated pathway. J Biol Chem 283:5127-5137.

Stone JS, Rubel EW (1999) Deltal expression during avian hair cell regeneration. Development 126:961-973.

Taylor RR, Filia A, Paredes U, Asai Y, Holt JR, Lovett M, Forge A (2018) Regenerating hair cells in vestibular sensory epithelia from humans. eLife 7:e34817.

Wang T, Chai R, Kim GS, Pham N, Jansson L, Nguyen DH, Kuo B, May LA, Zuo J, Cunningham LL, Cheng AG (2015) Lgr5 $^{+}$cells regenerate hair cells via proliferation and direct transdifferentiation in damaged neonatal mouse utricle. Nat Commun 6:6613.

Warchol ME (2007) Characterization of supporting cell phenotype in the avian inner ear: implications for sensory regeneration. Hear Res 227:11-18.

Warchol ME, Lambert PR, Goldstein BJ, Forge A, Corwin JT (1993) Regenerative proliferation in inner ear sensory epithelia from adult guinea pigs and humans. Science 259:1619-1622.

Wheelock MJ, Shintani Y, Maeda M, Fukumoto Y, Johnson KR (2008) Cadherin switching. J Cell Sci 121:727-735.

White PM, Stone JS, Groves AK, Segil N (2012) EGFR signaling is required for regenerative proliferation in the cochlea: conservation in birds and mammals. Dev Biol 363:191-200.

WHO Media Centre (2019) Deafness and hearing loss. Geneva: World Health Organisation.

Wibowo I, Pinto-Teixeira F, Satou C, Higashijima S, López-Schier H (2011) Compartmentalized notch signaling sustains epithelial mirror symmetry. Development 138:1143-1152.

Yamashita H, Oesterle EC (1995) Induction of cell proliferation in mammalian inner-ear sensory epithelia by transforming growth factor alpha and epidermal growth factor. Proc Natl Acad Sci U S A 92:3152-3155.

You D, Guo L, Li W, Sun S, Chen Y, Chai R, Li H (2018) Characterization of Wnt and Notch-responsive Lgr5 ${ }^{+}$hair cell progenitors in the striolar region of the neonatal mouse utricle. Front Mol Neurosci 11:137.

Zheng JL, Helbig C, Gao WQ (1997) Induction of cell proliferation by fibroblast and insulin-like growth factors in pure rat inner ear epithelial cell cultures. J Neurosci 17:216-226.

Zheng G, Lyons JG, Tan TK, Wang Y, Hsu T-T, Min D, Succar L, Rangan GK, Hu M, Henderson BR, Alexander SI, Harris DCH (2009) Disruption of E-Cadherin by Matrix Metalloproteinase Directly Mediates EpithelialMesenchymal Transition Downstream of Transforming Growth Factor- $\beta 1$ in Renal Tubular Epithelial Cells. Am J Pathol 175:580-591. 